\title{
A New Recursion in the Theory of Macdonald Polynomials
}

\author{
by \\ A. M. Garsia and J. Haglund
}

\begin{abstract}
.
The bigraded Frobenius characteristic of the Garsia-Haiman module $\mathbf{M}_{\mu}$ is known [7] [10] to be given by the modified Macdonald polynomial $\tilde{H}_{\mu}[X ; q, t]$. It follows from this that, for $\mu \vdash n$ the symmetric polynomial $\partial_{p_{1}} \tilde{H}_{\mu}[X ; q, t]$ is the bigraded Frobenius characteristic of the restriction of $\mathbf{M}_{\mu}$ from $S_{n}$ to $S_{n-1}$. The theory of Macdonald polynomials gives explicit formulas for the coefficients $c_{\mu \nu}$ occurring in the expansion $\partial_{p_{1}} \tilde{H}_{\mu}[X ; q, t]=\sum_{\nu \rightarrow \mu} c_{\mu \nu} \tilde{H}_{\nu}[X ; q, t]$. In particular it follows from this formula that the bigraded Hilbert series $F_{\mu}(q, t)$ of $\mathbf{M}_{\mu}$ may be calculated from the recursion $F_{\mu}(q, t)=\sum_{\nu \rightarrow \mu} c_{\mu \nu} F_{\nu}(q, t)$. One of the frustrating problems of the theory of Macdonald polynomials has been to derive from this recursion that $F_{\mu}(q, t) \in \mathbf{N}[q, t]$. This difficulty arises form the fact that the $c_{\mu \nu}$ have rather intricate expressions as rational functions in $q, t$. We give here a new recursion, from which a new combinatorial formula for $F_{\mu}(q, t)$ can be derived when $\mu$ is a two column partition. The proof suggests a method for deriving an analogous formula in the general case. The method was successfully carried out for the hook case by M. Yoo in [15].
\end{abstract}

\section{Introduction}

Let us recall that the Garsia-Haiman module $\mathbf{M}_{\mu}$ for $\mu \vdash n$ is defined in [7] as the linear span of the derivatives of the polynomial $\Delta_{\mu}(x, y)=\operatorname{det}\left\|x_{i}^{p_{j}} y_{i}^{q_{j}}\right\|_{i, j=1}^{n}$ where $\left(p_{1}, q_{1}\right),\left(p_{1}, q_{1}\right), \ldots,\left(p_{n}, q_{n}\right)$ are the cooordinates of the south-west corners of the cells of $\mu$. It was conjectured in [7] and proved in [10] that the bigraded Frobenius characteristic of $\mathbf{M}_{\mu}$ under the diagonal action of $S_{n}$ on the coordinates $x_{1}, x_{2}, \ldots, x_{n} ; y_{1}, y_{2}, \ldots, y_{n}$ is the modified Macdonald polynomial $\tilde{H}_{\mu}[X ; q, t]$. Denoting by $F_{\mu}(q, t)$ the bigraded Hilbert series of $\mathbf{M}_{\mu}$, it follows that we must have have

$$
F_{\mu}(q, t)=\partial_{p_{1}}^{n} \tilde{H}_{\mu}[X ; q, t] .
$$

Now it is shown in [6] that the Macdonald Pieri rules [11] yield

$$
\partial_{p_{1}} \tilde{H}_{\mu}[X ; q, t]=\sum_{\nu \rightarrow \mu} c_{\mu \nu}(q, t) \tilde{H}_{\nu}[X ; q, t]
$$

with

$$
c_{\mu \nu}(q, t)=\prod_{s \in \mathcal{R}_{\mu / \nu}} \frac{t^{l_{\mu}(s)}-q^{a_{\mu}(s)+1}}{t^{l_{\mu}(s)}-q^{a_{\mu}(s)}} \prod_{s \in \mathcal{C}_{\mu / \nu}} \frac{q^{a_{\mu}(s)}-t^{l_{\mu}(s)+1}}{q^{a_{\mu}(s)}-t^{l_{\mu}(s)}} .
$$

where $\mathcal{R}_{\mu / \nu}$ (resp. $\mathcal{C}_{\mu / \nu}$ ) denotes the set of lattice squares of $\nu$ that are in the same row (resp. same column) as the cell we must remove from $\mu$ to obtain $\nu$ and for any cell $s \in \mu$, the parameter $l_{\mu}(s)$ gives the number of cells of $\mu$ that are strictly north of $s$ and $a_{\mu}(s)$ gives the number of cells that are strictly east.

In particular, upon differentiation of both sides of I.2 by $\partial_{p_{1}}^{n-1}$, we derive that $F_{\mu}(q, t)$ may be computed from the recursion

$$
F_{\mu}[X ; q, t]=\sum_{\nu \rightarrow \mu} c_{\mu \nu}(q, t) F_{\nu}[X ; q, t]
$$


and initial condition $F_{(1)}(q, t)=1$. Although in [7] the nature of the coefficients $c_{\mu \nu}(q, t)$ was given a representation theoretical interpretation, their complexity, prevented obtaining any kind of explicit formula for $F_{\mu}(q, t)$ from I.4.

Being Hilbert series of finite dimensional modules the $F_{\mu}(q, t)$ are necessarily polynomials with positive integer coefficients. Thus a problem emerged form the onset of the Theory of Macdonald polynomials to obtain, a recursive construction of the $F_{\mu}(q, t)$ from which the combinatorics of these remarkable polynomials would emerge in a natural way, directly from the representation theoretical model.

Although both $\tilde{H}_{\mu}[X ; q, t]$ and (consequently) also $F_{\mu}(q, t)$ have recently been given purely combinatorial constructions [9], the latter were derived from an entirely manipulatorial path based on identities in the original Macdonald work [12], and their relation to the modules $\mathbf{M}_{\mu}$ is yet to be found.

In this paper we show that, at least in the two column case, a recursion may be given that stems right out of the representation theory of $\mathbf{M}_{\mu}$. This recursion may be stated as follows

$$
F_{2^{b}, 1^{a-b}}(q, t)=[b]_{t}(1+q) F_{2^{b-1}, 1^{a-b+1}}(q, t)+[a-b]_{t} t^{b} F_{2^{b}, 1^{a-b-1}}(q / t, t)
$$

It is quite straight forward to show that, I.5 is equivalent to the combinatorial formula

$$
F_{\mu}(q, t)=\sum_{T \in S T(\mu)} \prod_{i \in T}\left[d_{i}(T)\right]_{t} \times \prod_{i \in C_{2}(T)}\left(q+t^{b_{i}(T)}\right)
$$

where the sum is over all standard tableaux of shape $\mu=2^{b-1}, 1^{a-b+1}, d_{i}(T)$ is the number of rows of length equal to the length of the row of $i$ in the tableau obtained by removing from $T$ all the entries $j>i$, the second product is over the entries in the second column of $T$ and $b_{i}(T)$ denotes the number of entries $j>i$ in the first column of $T$.

Remarkably, it turns out that I.5 is only the tip of an iceberg. Indeed, the underlying representation theoretical identity is none other than the Frobenius characteristic recursion

$$
\partial_{p_{1}} \tilde{H}_{2^{b}, 1^{a-b}}(q, t)=[b]_{t}(1+q) \tilde{H}_{2^{b-1}, 1^{a-b+1}}(q, t)+[a-b]_{t} t^{b} \tilde{H}_{2^{b}, 1^{a-b-1}}(q / t, t)
$$

from which I.6 immediately follows upon differentiation of both sides by $\partial_{p_{1}}^{a+b-1}$.

The main result in this paper is a proof of I.7. In final analysis, our argument again only uses identities of the theory of Macdonald polynomials and could be presented in this manner without much further ado. However, this type of treatement would leave the reader puzzled as to how we could come up with the various manipulations we carry out to achieve the final result. For this reason and for the benefit future research aimed at finding an analogous formula in the general case, we will carefully go over the representation theoretical reasoning that guided every step of our argument. At the end we will also explore in detail the case of $\mu=(3,2,1)$ as evidence suggesting that the same representation theoretical reasoning can be carried out in full generality. We should mention that the hook case was carried out in the PhD thesis of M. Yoo [15].

Our developments here rely heavily on the contents of [1] and [5]. In particular, we will show that I.7 can be beautifully imbedded into the "Science Fiction" heuristics introduced in [1]. In fact, we will see that I.7 adds a new twist into the "Science Fiction" of the modules $\mathbf{M}_{\mu}$. This given, the reader is urged 
to have at hand at least [1], since for brevity, we will have to assume some familiarity with the results and notation introduced there.

1. The case $a=3$ and $b=2$.

It will be good to represent Macdonald polynomials by Ferrers diagrams. This given 1.2 becomes

$$
\partial_{p_{1}} \boldsymbol{由}_{(q, t)}=(1+t)(1+q) \text { 且 }(q, t)+\boldsymbol{⿴ 囗 十 ~}_{(q / t, t) t^{2}}
$$

Let us see then how we can interpret this identity using the Science Fiction model. Our point of departure is the determinant

$$
\Delta_{\text {田 }}=\operatorname{det}\left[\begin{array}{ccccc}
1 & 1 & 1 & 1 & 1 \\
y_{1} & y_{2} & y_{3} & y_{4} & y_{5} \\
x_{1} & x_{2} & x_{3} & x_{4} & x_{5} \\
x_{1} y_{1} & x_{2} y_{2} & x_{3} y_{3} & x_{4} y_{4} & x_{5} y_{5} \\
x_{1}^{2} & x_{2}^{2} & x_{3}^{2} & x_{4}^{2} & x_{5}^{2}
\end{array}\right]
$$

with expansion

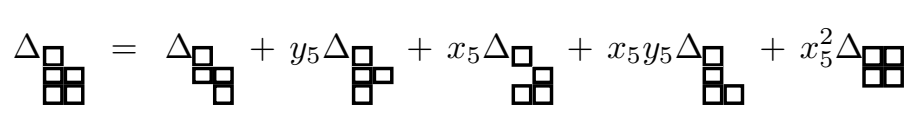

This gives

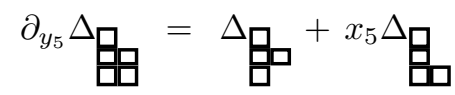

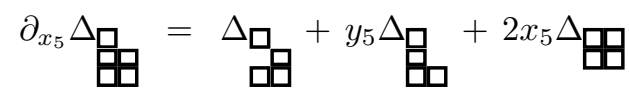

$$
\begin{aligned}
& \partial_{x_{5}} \partial_{y_{5}} \Delta_{\boldsymbol{H}}={ }^{\Delta_{\text {日 }}} \\
& \partial_{x_{5}}^{2} \Delta_{\text {田 }}={ }^{2 \Delta} \text { 由 }
\end{aligned}
$$

To construct a basis for the linear span of derivatives of $\Delta_{\text {g }}$, we will seek for 5 collections of monomials in the alphabet $x_{1}, x_{2}, \ldots, x_{4} ; y_{1}, y_{2}, \ldots, y_{4}$

$$
\mathcal{B}_{00}, \mathcal{B}_{01}, \mathcal{B}_{10}, \mathcal{B}_{11}, \mathcal{B}_{20}
$$

such that the collections

$$
\begin{aligned}
& \left\{b_{00}(\partial) \Delta_{\boldsymbol{~}}\right\}_{b_{00} \in \mathcal{B}_{00}},\left\{b_{01}(\partial) \partial_{y_{5}} \Delta_{\boldsymbol{B}}\right\}_{b_{01} \in \mathcal{B}_{01}},\left\{b_{10}(\partial) \partial_{x_{5}} \Delta_{\boldsymbol{中}}\right\}_{b_{10} \in \mathcal{B}_{10}}, \\
& \left\{b_{11}(\partial) \partial_{x_{5}} \partial_{y_{5}} \Delta \boldsymbol{\boxplus}\right\}_{b_{11} \in \mathcal{B}_{11}},\left\{b_{20}(\partial) \partial_{x_{5}}^{2} \Delta \boldsymbol{\boxplus}\right\}_{b_{20} \in \mathcal{B}_{20}},
\end{aligned}
$$

form a basis for the module $\mathbf{M}_{32}$. The collections $\mathcal{B}_{i j}$ must be determined so that the cardinality of their union is 5 ! and for any choices of $b_{i j} \in \mathcal{B}_{i j}$ the identity

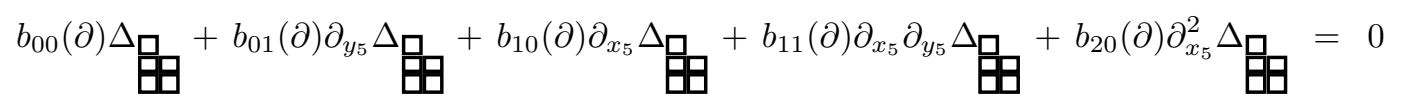


forces

$$
b_{00}=b_{01}=b_{10}=b_{11}=b_{20}=0
$$

Now using the identities in $1.3-1.7$ the equality in 1.8 converts to

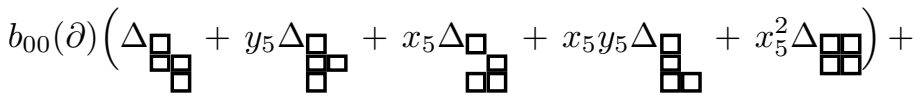

$$
\begin{aligned}
& b_{01}(\partial)\left(\Delta_{\boldsymbol{g}}+x_{5} \Delta_{\boldsymbol{D}}\right)+ \\
& b_{10}(\partial)\left(\Delta_{\boldsymbol{H}}+y_{5} \Delta_{\boldsymbol{B}}+2 x_{5} \Delta_{\mathbf{H}}\right)+ \\
& b_{11}(\partial) \Delta_{\text {日 }}+ \\
& 2 b_{20}(\partial) \Delta_{\boldsymbol{B}}=0
\end{aligned}
$$

equating to zero the terms independent of $x_{5}, y_{5}$ and equating the coefficients of $y_{5}, x_{5}, x_{5} y_{5}$ and $x_{5}^{2}$ yields the system

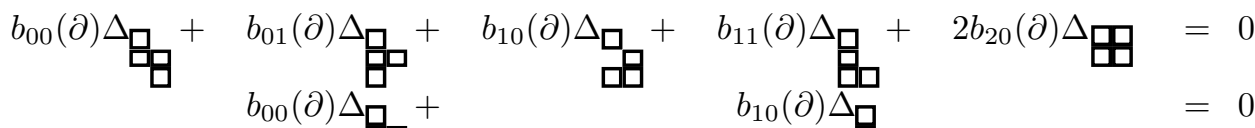

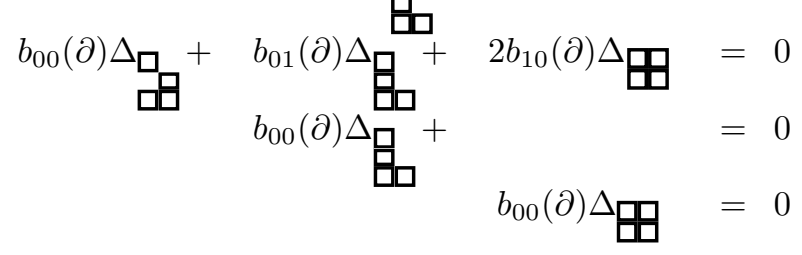

Note that the last two equations say that $b_{00}(\partial)$ kills both ${ } \boldsymbol{B}$ and $\boldsymbol{\Delta}^{\text {this given these two equations will }}$ force $b_{00}$ to vanish identically if $\mathcal{B}_{00}$ is chosen to be a basis for the subspace $\mathbf{M} \vee \mathbf{M}{ }^{\text {of }} \mathbf{M}_{\boldsymbol{g}}$. We shall represent this by writing

$$
\mathbf{B}_{00}=\text { 且 } \vee \text { }
$$

with this choice of $\mathcal{B}_{00} 1.9$ reduces to

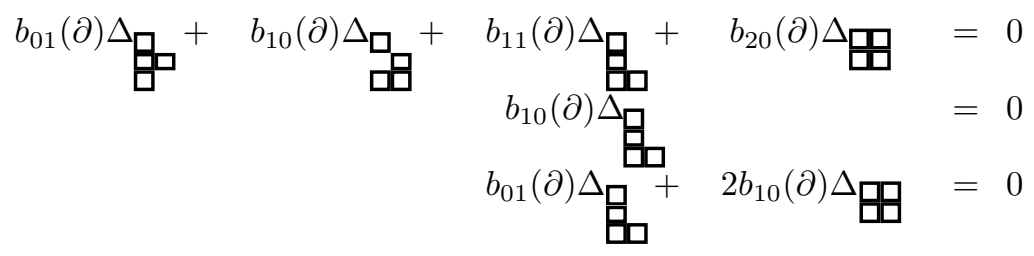

Now the second equation will force $b_{10}=0$ if we set (using the notation in 1.10)

$$
\mathcal{B}_{10}=\text { 旦 }
$$


with this choice of $\mathcal{B}_{10} 3.11$ reduces to

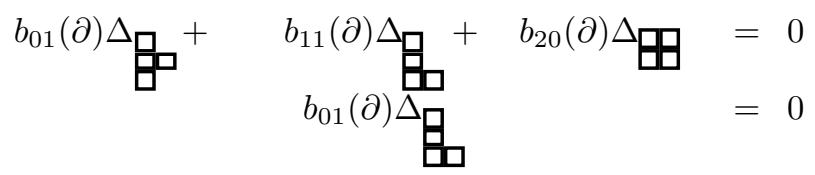

and the second equation here will force the vanishing of $b_{10}$ if we set

$$
\mathcal{B}_{10}=\text { 旦 }
$$

with this choice of $\mathcal{B}_{10} 1.13$ reduces to

$$
{ }^{b_{11}(\partial) \boldsymbol{H}}+{ }^{b_{20}(\partial) \Delta} \boldsymbol{\boxplus}=0
$$

At this point we will choose

$$
\mathcal{B}_{11}=\text { 旦 }
$$

To guarantee that 1.15 will force the vanishing of $b_{10}$ we need only assure that the term " $b_{20}(\partial) \Delta$ 田" will not produce an element of $\mathbf{M}_{\text {B }}$. Now Science Fiction states that if

$$
\mathcal{B}_{20}=\text { 且 } \wedge \text { 田 }
$$

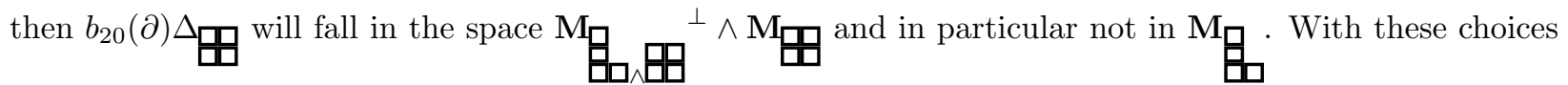
let us now compute the cardinalities of the resulting collections. In fact, in order to derive the implications of 1.1 it will be informative to compute the Frobenius characterists of the spaces spanned by the chosen collections. From Science Fiction again we know that in this case every Frobenius Characteristic can be expressed in term of the Frobenius Characteristic of the $\mathcal{S}_{4}$ module $\mathbf{M}_{\boldsymbol{B}} \wedge \mathrm{M}_{\mathbf{B}}$. Denoting by "F" the Frobenius map, Science Fiction gives

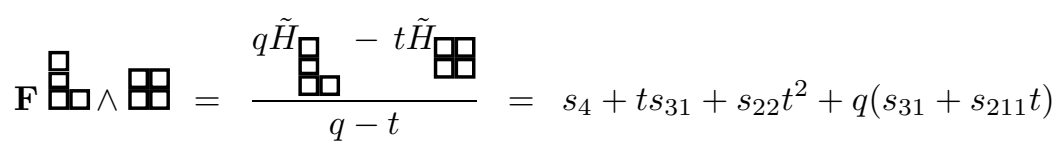

Denoting this symmetric function by $\phi_{32}(q, t ; x)$ we derive that (again using Science Fiction)

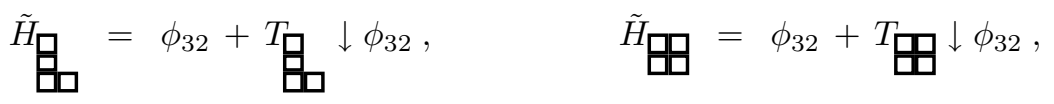

where for a symmetric polynomial $P$ with coefficients in $\mathbb{Q}(q, t)$ we set

$$
\downarrow P=\left.\omega P\right|_{t \rightarrow \frac{1}{t}, q \rightarrow \frac{1}{q}}
$$


Since $T_{\text {日 }}=t^{3} q$ and $T \mathbb{\boxplus}=t^{2} q^{2}$ our choices then give

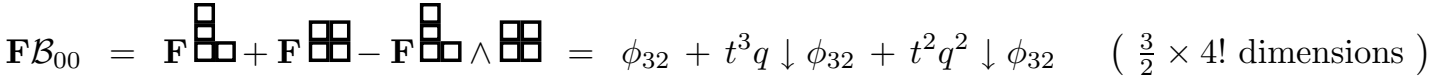

$$
\begin{aligned}
& \mathbf{F} \mathcal{B}_{01}=\tilde{H}_{\text {且 }}=\phi_{32}+t^{3} q \downarrow \phi_{32} \quad(4 \text { ! dimensions }) \\
& \mathbf{F B}_{10}=\tilde{H}_{\text {且 }}=\phi_{32}+t^{3} q \downarrow \phi_{32} \quad(4 \text { ! dimensions }) \\
& \mathbf{F B}_{11}=\tilde{H}_{\text {且 }}=\phi_{32}+t^{3} q \downarrow \phi_{32} \quad(4 \text { ! dimensions }) \\
& \mathbf{F B}_{20}=\mathbf{F} \text { 旦} \wedge \boldsymbol{H}=\phi_{32} \quad\left(\frac{1}{2} \times 4 \text { ! dimensions }\right)
\end{aligned}
$$

The Frobenius characteristic of the modules spanned by the collections in $\left(^{*}\right)$ is thus

$$
{ }^{T} \text { 田 }{ }^{\downarrow}\left((1+t)(1+q) \tilde{H}_{\text {是 }}+t^{2} q^{2} \downarrow \phi_{32}+t^{2} \phi_{32}\right)
$$

or

$$
t^{4} q^{2}\left((1+1 / t)(1+1 / q) \downarrow \tilde{H}_{\mathbf{H}}+1 / t^{2} q^{2} \phi_{32}+1 / t^{2} \downarrow \phi_{32}\right)
$$

since $t^{3} q \downarrow \tilde{H}_{\text {日 }}=\tilde{H}_{\text {日 }} 1.20$ becomes

$$
(1+t)(1+q) \tilde{H}_{\mathbf{H}^{2}}+t^{2} \phi_{32}+t^{2} q^{2} \downarrow \phi_{32}
$$

In conclusion Science Fiction gives

$$
\partial_{p_{1}} \tilde{H}_{\text {早 }}=(1+t)(1+q) \tilde{H}_{\theta}+t^{2} \phi_{32}+t^{2} q^{2} \downarrow \phi_{32}
$$

Now recall that the recursion in I.7 for $a=3$ and $b=2$ is

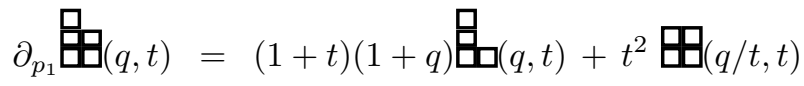

we see that to understand this identity we need only explain why

$$
t^{2} \phi_{32}+t^{2} q^{2} \downarrow \phi_{32}=t^{2} \tilde{H}_{\mathbf{B}^{(q / t, t)} .}
$$

Now recall that we have the direct sum decomposition

$$
{ }^{M_{\boxplus}}=\mathrm{M}_{\boldsymbol{B}} \wedge \mathrm{M}_{\boxplus} \oplus\left(\mathrm{M}_{\boldsymbol{B}} \wedge \mathrm{M}_{\boldsymbol{B}}\right)^{\perp} \wedge \mathrm{M}_{\boldsymbol{B}}
$$


which combined with Science Fiction yields that

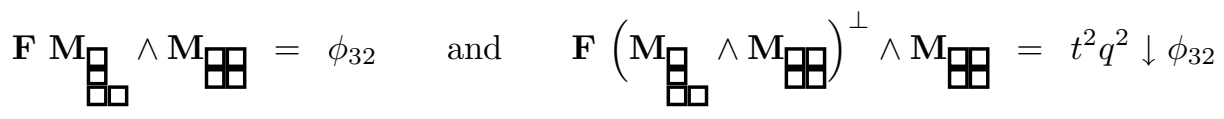

causing the identity

$$
\tilde{H}_{\boldsymbol{H}}=\phi_{32}+t^{2} q^{2} \downarrow \phi_{32}
$$

In summary we must understand why the replacement $\phi_{32} \rightarrow t^{2} \phi_{32}$ in this identity can be achieved by the substitution $q \rightarrow q / t$ in $\tilde{H}_{\mathbf{B}}$ followed by multiplication by $t^{2}$. Note that classically we used to write

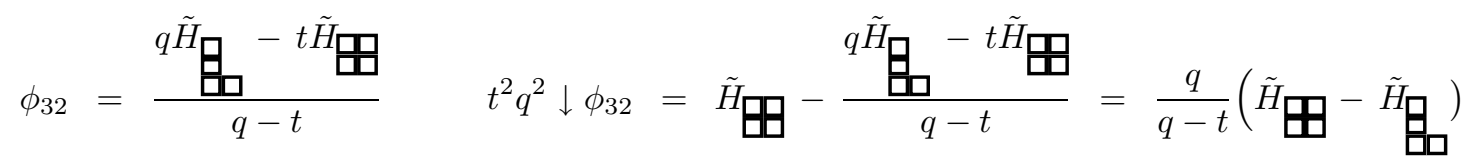

which gave that

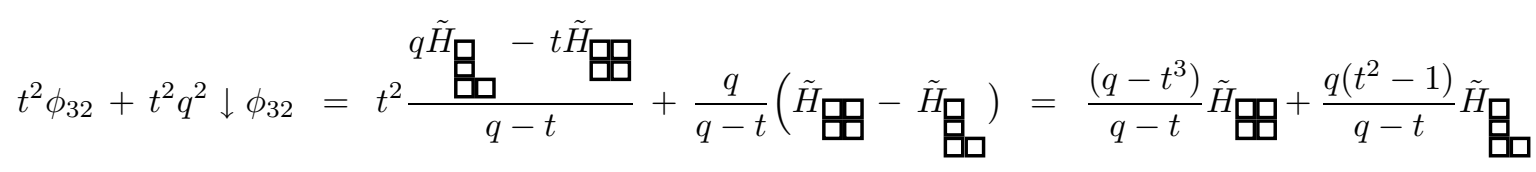

and this was responsible for the rationality of the recursion we had before (in terms of the dual Pieri " $c_{\mu \nu}{ }^{\prime} s$ ). Now we see that 1.21 is an incredibly simple way of avoiding the rationalities that have pestered us for so many years!.

The explanation of 1.21 comes from a totally unexpected source: "k-schurs"! Indeed it follows from the Morse-Lapointe-Lascoux theory [11] that all our ingredients in 1.21 are in the linear span of the 2-schur "atoms"

$$
A_{2,2}=s_{4}+t s_{3,1}+t^{2} s_{2,2}, \quad A_{2,1,1}=s_{31}+t s_{2,1,1}, \quad A_{1,1,1,1}=s_{2,2}+t s_{2,1,1}+t^{2} s_{1,1,1,1}
$$

For instance we have

$$
\tilde{H}_{\mathbf{⿴ 囗 十 ~}}=A_{2,2}+q A_{2,1,1}+q t A_{2,1,1}+q^{2} A_{1,1,1,1}
$$


or in matrix representation $(\dagger)$

$$
\tilde{H}_{\boldsymbol{G}}=\left[\begin{array}{ccc}
0 & A_{211} & 0 \\
A_{22} & A_{211} & A_{1111}
\end{array}\right]
$$

and

$$
\phi_{32}=A_{2,2}+q A_{2,1,1}, \quad t^{2} q^{2} \downarrow \phi_{32}=q t A_{2,1,1}+q^{2} A_{1111}
$$

thus

$$
t^{2} \phi_{32}=\left[\begin{array}{cc}
A_{22} & A_{211} \\
0 & 0 \\
0 & 0
\end{array}\right], \quad t^{2} q^{2} \downarrow \phi_{32}=\left[\begin{array}{ccc}
0 & A_{211} & 0 \\
0 & 0 & A_{1111}
\end{array}\right]
$$

so

$$
t^{2} \phi_{32}+t^{2} q^{2} \downarrow \phi_{32}=\left[\begin{array}{ccc}
A_{22} & A_{211} & 0 \\
0 & A_{211} & 0 \\
0 & 0 & A_{1111}
\end{array}\right]
$$

Now from 1.24 we derive that

$$
t^{2} \tilde{H}_{\mathbf{G}}(q / t, t)=t^{2} A_{2,2}+t q\left(A_{2,1,1}+t A_{2,1,1}\right)+q^{2} A_{1,1,1,1}
$$

what has happened is that the two atoms $A_{2,2}, A_{2,1,1}$ which where in the bottom row of $\tilde{H}_{\boldsymbol{B}}$ have been lifted differently (the first to the $3 r d$ row and the second to the end row). Moreover the atom $A_{1,1,1,1}$ remained unlifted. This permits a recombination of atoms causing the left hand side of 1.21 to be equal to the right hand side. This recombination is also clearly visible from the manipulations below

$$
\begin{aligned}
t^{2} \phi_{32}+t^{2} q^{2} \downarrow \phi_{32} & =t^{2}\left(A_{2,2}+q A_{2,1,1}\right)+\left(q t A_{2,1,1}+q^{2} A_{1111}\right) \\
& =t^{2} A_{2,2}+\left(t^{2} q A_{2,1,1}+q t A_{2,1,1}\right)+q^{2} A_{1111} \\
& =t^{2} A_{2,2}+t q\left(A_{2,1,1}+t A_{2,1,1}\right)+q^{2} A_{1111} \\
& =t^{2} \tilde{H}_{\mathbf{G}}(q / t, t)
\end{aligned}
$$

In the next section we will use another heuristic that will substantially short cut the derivation of the general form of the latter identity.

\section{The general 2-column case, with kicking identities}

The point of departure here are the two kicking identities [6], (see also [4]).

$$
\begin{aligned}
& \partial_{p_{1}} \tilde{H}_{2^{b} 1^{a-b}}=[a-b]_{t} \text { 苖 }+t^{a-b}[b]_{t} \text { 昌 }+q[b]_{t} \text { 苖 } \\
& \partial_{p_{1}} \tilde{H}_{2^{b} 1^{a-b}}=t^{b}[a-b]_{t} \text { 昌 }_{q}{ }_{q}[b]_{t} \text { 甼 }+[b]_{t} \text { 甼 }
\end{aligned}
$$

$(\dagger)$ We use the matrix $\left\|c_{i j}\right\|_{\substack{0 \leq i \leq h \\ 0 \leq j \leq k}}$ to represent the polynomial $P(q, t)=\sum_{i=0}^{h} \sum_{j=0}^{k} c_{i j} t^{i} q^{j}$ 
where for convenience we have used the notation

$$
\tilde{H}_{2^{b} 1^{a-b-1}}=\text { 田, } \tilde{H}_{2^{b-1} 1^{a-b+1}}=\text { 困 }
$$

and the symbol " $由$ ", represents a Frobenius characteristic to be determined from 2.1 and 2.2 In fact subtracting 2.2 from 2.1 we get

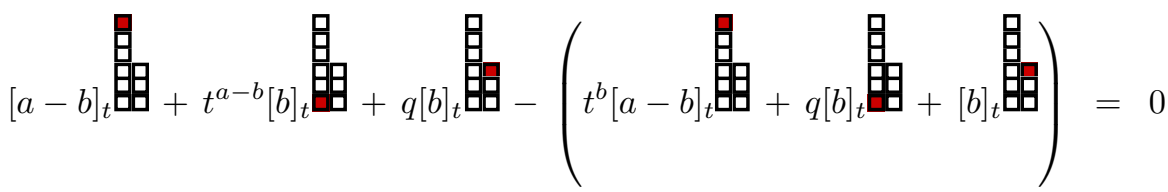

$$
\begin{aligned}
& \left(t^{a-b}[b]_{t}-q[b]_{t}\right) \text { 苖 }=\left(t^{b}-1\right)[a-b]_{t} \text { 苖 }+[b]_{t}(1-q) \text { 苖 }
\end{aligned}
$$

using $[b]_{t}=\left(1-t^{b}\right) /(1-t)$ and $[a-b]_{t}=\left(1-t^{a-b}\right) /(1-t)$ and cancelling $[b]_{t}$ this becomes

$$
\left(t^{a-b}-q\right) \text { 困 }=\left(t^{a-b}-1\right) \text { 困 }+(1-q) \text { 田 }
$$

yielding

$$
\text { 困 }=\frac{\left(t^{a-b}-1\right) \text { 甼 }+(1-q) \text { 困 }}{t^{a-b}-q}
$$

Now Science Fiction gives

$$
\text { 田 }=\phi_{a b}+t^{\left(\begin{array}{c}
a-1 \\
2
\end{array}\right)+\left(\begin{array}{c}
b \\
2
\end{array}\right)} q^{b} \downarrow \phi_{a b}, \quad \text { 田 }=\phi_{a b}+t^{\left(\begin{array}{c}
a \\
2
\end{array}\right)+\left(\begin{array}{c}
b-1 \\
2
\end{array}\right) q^{b-1} \downarrow \phi_{a b}}
$$

where

$$
\phi_{a b}=\mathbf{F} \text { 田昌 }
$$

Using 2.4 in 2.3 gives

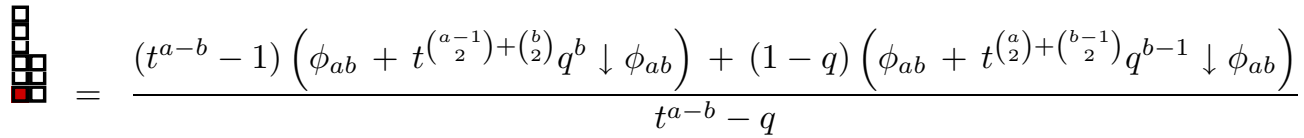

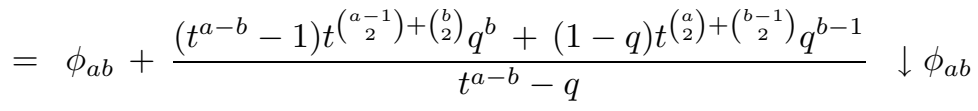




$$
\begin{aligned}
& =\phi_{a b}+t^{\left(\begin{array}{c}
a-1 \\
2
\end{array}\right)+\left(\begin{array}{c}
b-1 \\
2
\end{array}\right)} q^{b-1} \frac{\left(t^{a-b}-1\right) t^{b-1} q+(1-q) t^{a-1}}{t^{a-b}-q} \downarrow \phi_{a b} \\
& =\phi_{a b}+t^{\left(\begin{array}{c}
a-1 \\
2
\end{array}\right)+\left(\begin{array}{c}
b-1 \\
2
\end{array}\right)} q^{b-1} \frac{t^{a-1} q-t^{b-1} q+t^{a-1}-q t^{a-1}}{t^{a-b}-q} \downarrow \phi_{a b} \\
& =\phi_{a b}+t^{\left(\begin{array}{c}
a-1 \\
2
\end{array}\right)+\left(\begin{array}{c}
b-1 \\
2
\end{array}\right)} q^{b-1} \frac{-t^{b-1} q+t^{a-1}}{t^{a-b}-q} \downarrow \phi_{a b} \\
& =\phi_{a b}+t^{\left(\begin{array}{c}
a-1 \\
2
\end{array}\right)+\left(\begin{array}{c}
b-1 \\
2
\end{array}\right)} q^{b-1} t^{b-1} \downarrow \phi_{a b}
\end{aligned}
$$

In summary

$$
\text { 昌 }=\phi_{a b}+t^{\left(\begin{array}{c}
a-1 \\
2
\end{array}\right)+\left(\begin{array}{l}
b \\
2
\end{array}\right) q^{b-1} \downarrow \phi_{a b}}
$$

We now use 2.4 and 2.6 in 2.2 and obtain

$$
\begin{aligned}
& \partial_{p_{1}} \tilde{H}_{2^{b} 1^{a-b}}= t^{b}[a-b]_{t}\left(\phi_{a b}+t^{\left.\left(\begin{array}{c}
a-1 \\
2
\end{array}\right)+\left(\begin{array}{c}
b \\
2
\end{array}\right) q^{b} \downarrow \phi_{a b}\right)}\right. \\
&+q[b]_{t}\left(\phi_{a b}+t^{\left(\begin{array}{c}
a-1 \\
2
\end{array}\right)+\left(\begin{array}{c}
b \\
2
\end{array}\right)} q^{b-1} \downarrow \phi_{a b}\right)+[b]_{t}\left(\phi_{a b}+t^{\left.\left(\begin{array}{c}
a \\
2
\end{array}\right)+\left(\begin{array}{c}
b-1 \\
2
\end{array}\right) q^{b-1} \downarrow \phi_{a b}\right)}\right. \\
&=\left(t^{b}[a-b]_{t}+q[b]_{t}+[b]_{t}\right) \phi_{a b} \\
& \quad+t^{\left(\begin{array}{c}
a-1 \\
2
\end{array}\right)+\left(\begin{array}{c}
b-1 \\
2
\end{array}\right)} q^{b-1}\left(t^{b}[a-b]_{t} t^{b-1} q+q[b]_{t} t^{b-1}+[b]_{t} t^{a-1}\right) \downarrow \phi_{a b} \\
&=\left(t^{b}[a-b]_{t}+q[b]_{t}+[b]_{t}\right) \phi_{a b} \\
& \quad+t^{\left(\begin{array}{c}
a-1 \\
2
\end{array}\right)+\left(\begin{array}{c}
b-1 \\
2
\end{array}\right)} q^{b-1} t^{b-1}\left(t^{b}[a-b]_{t} q+q[b]_{t}+[b]_{t} t^{a-b}\right) \downarrow \phi_{a b}
\end{aligned}
$$

We thus obtain

$$
\partial_{p_{1}} \tilde{H}_{2^{b} 1^{a-b}}=\left([a]_{t}+q[b]_{t}\right) \phi_{a b}+t^{\left(\begin{array}{c}
a-1 \\
2
\end{array}\right)+\left(\begin{array}{c}
b-1 \\
2
\end{array}\right)} q^{b-1} t^{b-1}\left([a]_{t} q+[b]_{t} t^{a-b}\right) \downarrow \phi_{a b}
$$

As a check note that for $a=3$ and $b=2$ we get

$$
\begin{aligned}
\partial_{p_{1}} \tilde{H}_{221} & =\left([3]_{t}+q(1+t)\right) \phi_{3,2}+q^{1} t^{2}\left([3]_{t} q+(1+t) t^{1}\right) \downarrow \phi_{3,2} \\
& =\left([3]_{t}+q(1+t)\right) \phi_{3,2}+\left([3]_{t} t^{2} q^{2}+(1+t) t^{3} q\right) \downarrow \phi_{3,2} \\
& =t^{2} \phi_{3,2}+(1+q)(1+t) \phi_{3,2}+\left(t^{2} q^{2}+t^{3} q^{2}+t^{4} q^{2}+(1+t) t^{3} q\right) \downarrow \phi_{3,2} \\
& =(1+q)(1+t) \phi_{3,2}+(q+t q+(1+t)) t^{3} q \downarrow \phi_{3,2}+t^{2} \phi_{3,2}+t^{2} q^{2} \downarrow \phi_{3,2} \\
& =(1+q)(1+t) \phi_{3,2}+(1+q)(1+t) t^{3} q \downarrow \phi_{3,2}+t^{2} \phi_{3,2}+t^{2} q^{2} \downarrow \phi_{3,2} \\
& =(1+q)(1+t) \tilde{H}_{211}+t^{2} \phi_{3,2}+t^{2} q^{2} \downarrow \phi_{3,2}
\end{aligned}
$$

This is what we previously had using only Science Fiction and we saw that a comparison with 1.1 yielded the identity

$$
t^{2} \phi_{3,2}+T_{\boldsymbol{B}} \downarrow \phi_{3,2}=t^{2} \boldsymbol{\theta}(q / t, t)
$$

It turns out that an entirely analogous result holds true in full generality. More precisely we will show that 


\section{Theorem 2.1}

For all $a>b$ the recursion in 1.1 is equivalent to the identity

$$
t^{b} \phi_{a, b}+T_{\text {是 }} \downarrow \phi_{a, b}=t^{b} \text { 苖 }(q / t, t)
$$

\section{Proof}

Our point of deparure is the identity in 2.7 which combined with 1.2 implies that

$$
\begin{aligned}
& \left([a]_{t}+q[b]_{t}\right) \phi_{a b}+t^{\left(\begin{array}{c}
a-1 \\
2
\end{array}\right)+\left(\begin{array}{c}
b-1 \\
2
\end{array}\right)} q^{b-1} t^{b-1}\left([a]_{t} q+[b]_{t} t^{a-b}\right) \downarrow \phi_{a b}= \\
& =[b]_{t}(1+q) \tilde{H}_{2^{b-1}, 1^{a-b+1}}(q, t)+[a-b]_{t} t^{b} \tilde{H}_{2^{b}, 1^{a-b-1}}(q / t, t)
\end{aligned}
$$

or better, using 2.4 and making the substitutions $[a]_{t} \rightarrow[b]_{t}+t^{b}[a-b]_{t}$ and $a-1+\left(\begin{array}{c}a-1 \\ 2\end{array}\right) \rightarrow\left(\begin{array}{c}a \\ 2\end{array}\right), b-1+\left(\begin{array}{c}b-1 \\ 2\end{array}\right) \rightarrow\left(\begin{array}{c}b \\ 2\end{array}\right)$

$$
\begin{aligned}
& \left([b]_{t}+t^{b}[a-b]_{t}+q[b]_{t}\right) \phi_{a b}+\left([a]_{t} t\left(\begin{array}{c}
a-1 \\
2
\end{array}\right)+\left(\begin{array}{c}
b \\
2
\end{array}\right) q^{b}+[b]_{t} t\left(\begin{array}{c}
a \\
2
\end{array}\right)+\left(\begin{array}{c}
b-1 \\
2
\end{array}\right) q^{b-1}\right) \downarrow \phi_{a b}= \\
& =[b]_{t}(1+q)\left(\phi_{a b}+t^{\left(\begin{array}{c}
a \\
2
\end{array}\right)+\left(\begin{array}{c}
b-1 \\
2
\end{array}\right)} q^{b-1} \downarrow \phi_{a b}\right)+[a-b]_{t} t^{b} \tilde{H}_{2^{b}, 1^{a-b-1}}(q / t, t)
\end{aligned}
$$

We will now proceed to eliminate equal terms from both sides until we are left with 2.8. This gives

$$
\begin{aligned}
& t^{b}[a-b]_{t} \phi_{a b}+[a]_{t} t^{\left(\begin{array}{c}
a-1 \\
2
\end{array}\right)+\left(\begin{array}{l}
b \\
2
\end{array}\right)} q^{b} \downarrow \phi_{a b}+[b]_{t} t^{\left(\begin{array}{c}
a \\
2
\end{array}\right)+\left(\begin{array}{c}
b-1 \\
2
\end{array}\right)} q^{b-1} \downarrow \phi_{a b}= \\
& =[b]_{t} t^{\left(\begin{array}{c}
a \\
2
\end{array}\right)+\left(\begin{array}{c}
b-1 \\
2
\end{array}\right)} q^{b-1} \downarrow \phi_{a b}+[b]_{t} t^{\left(\begin{array}{c}
a \\
2
\end{array}\right)+\left(\begin{array}{c}
b-1 \\
2
\end{array}\right)} q^{b} \downarrow \phi_{a b}+[a-b]_{t} t^{b} \tilde{H}_{2^{b}, 1^{a-b-1}}(q / t, t)
\end{aligned}
$$

and using $[a]_{t}=[a-b]_{t}+t^{a-b}[b]_{t}$

$$
\begin{aligned}
& t^{b}[a-b]_{t} \phi_{a b}+[a-b]_{t} t^{\left(\begin{array}{c}
a-1 \\
2
\end{array}\right)+\left(\begin{array}{c}
b \\
2
\end{array}\right)} q^{b} \downarrow \phi_{a b}+t^{a-b}[b]_{t} t^{\left(\begin{array}{c}
a-1 \\
2
\end{array}\right)+\left(\begin{array}{c}
b \\
2
\end{array}\right)} q^{b} \downarrow \phi_{a b}= \\
& =[b]_{t} t^{\left(\begin{array}{c}
a \\
2
\end{array}\right)+\left(\begin{array}{c}
b-1 \\
2
\end{array}\right)} q^{b} \downarrow \phi_{a b}+[a-b]_{t} t^{b} \tilde{H}_{2^{b}, 1^{a-b-1}}(q / t, t)
\end{aligned}
$$

and using $a-b+\left(\begin{array}{c}a-1 \\ 2\end{array}\right)+\left(\begin{array}{l}b \\ 2\end{array}\right)=\left(\begin{array}{l}a \\ 2\end{array}\right)+\left(\begin{array}{c}b-1 \\ 2\end{array}\right)$

$$
\begin{aligned}
& t^{b}[a-b]_{t} \phi_{a b}+[a-b]_{t} t^{\left(\begin{array}{c}
a-1 \\
2
\end{array}\right)+\left(\begin{array}{c}
b \\
2
\end{array}\right)} q^{b} \downarrow \phi_{a b}+[b]_{t} t^{\left(\begin{array}{c}
a \\
2
\end{array}\right)+\left(\begin{array}{c}
b-1 \\
2
\end{array}\right)} q^{b} \downarrow \phi_{a b}= \\
& =[b]_{t} t^{\left(\begin{array}{c}
a \\
2
\end{array}\right)+\left(\begin{array}{c}
b-1 \\
2
\end{array}\right)} q^{b} \downarrow \phi_{a b}+[a-b]_{t} t^{b} \tilde{H}_{2^{b}, 1^{a-b-1}}(q / t, t)
\end{aligned}
$$

and we are left with

$$
t^{b}[a-b]_{t} \phi_{a b}+[a-b]_{t} t^{\left(\begin{array}{c}
a-1 \\
2
\end{array}\right)+\left(\begin{array}{c}
b \\
2
\end{array}\right)} q^{b} \downarrow \phi_{a b}=[a-b]_{t} t^{b} \tilde{H}_{2^{b}, 1^{a-b-1}}(q / t, t)
$$

and this is 2.8 after cancelling the common factor $[a-b]_{t}$.

It is interesting to see how 2.8 for $a=4$ and $b=2$ follows by means of the Morse-Lapointe 2-atoms. In this case 2.8 reduces to

$$
t^{2} \phi_{4,2}+t^{4} q^{2} \downarrow \phi_{4,2}=t^{2} \tilde{H}_{221}(q / t, t)
$$


Now the 2-atom expansions of $\phi_{4,2}$ and $\tilde{H}_{221}(q, t)$ are
a) $\phi_{4,2}=A_{2111}+q A_{221}$,
b) $\left.\quad \tilde{H}_{221}(q, t)=A_{221}+(q+t q) A_{2111}+q^{2} A_{11111} b\right)$

and the identity $\tilde{H}_{221}(q, t)=\phi_{4,2}+t^{4} q^{2} \downarrow \phi_{4,2}$ gives

$$
t^{4} q^{2} \downarrow \phi_{4,2}=t q A_{2111}+q^{2} A_{11111}
$$

In matrix notation $2.9 \mathrm{~b}$ ) becomes

$$
\tilde{H}_{221}(q, t)=\left[\begin{array}{ccc}
0 & A_{2111} & 0 \\
A_{221} & A_{2111} & A_{11111}
\end{array}\right]
$$

where we can distinctly see both $\phi_{42}$ and $t^{4} q^{2} \downarrow \phi_{4,2}$. Now raising $\phi_{42}$ up two levels upon multiplication by $t^{2}$ yields the matrix

$$
\left[\begin{array}{ccc}
A_{221} & A_{2111} & 0 \\
0 & A_{2111} & 0 \\
0 & 0 & A_{11111}
\end{array}\right]=t^{2} \tilde{H}_{221}(q / t, t)
$$

which is exactly the same as raising the first column in 2.11 up two steps, the second column up one step and the third column up zero steps, which is precisely what the substitution $q \rightarrow q / t$ followed by multiplication by $t^{2}$ does on the matrix of $\tilde{H}_{[2,2,1]}$. We can thus clearly see that 2.8 is a new phenomenon to be added to Science Fiction! More precisely we see that certain "irreducible" submodules occurring in the Science Fiction decomposition of the modules $\mathbf{M}_{\mu}$ may be recombined into new submodules whose Frobenius characteristics are suitable combinatorial deformations of the Macdonald polynomials $\tilde{H}_{\mu}(x ; q, t)$. It just happens that when $\mu$ is a two column partition, this deformation takes the simple form $t^{k} \tilde{H}_{\mu}(X ; q / t, t)$. Later examples strongly suggest that this recombination of irreducibles is a general phenomenon.

The challenge is to see in which manner the Frobenius recursion in I.2 can be replaced by a recursion in which the terms $c_{\mu \nu} \tilde{H}_{\nu}(X ; q, t)$ are replaced by terms where $c_{\mu \nu}$ is replaced by a polynomial in $\mathbf{N}[q, t]$ and $\tilde{H}_{\nu}(X ; q, t)$ is replaced by a deformed version.

\section{The Science Fiction-less derivation of the equivalent symmetric function identity .}

Since, to this moment, most of Science Fiction is still conjectural, we will avoid in the next two sections to make any direct use of it. Nevertheless we must acknowledge that this proof I.7 would not have been possible without the guidance provided by Science Fiction heuristics.

The proof will consist of two separed parts. In the first part we will manipulatorially transform the equality in I.7 into an equivalent simpler identity by eliminating terms that are common to both sides. In the second part we give a proof of the simpler identity. We should mention that Science fiction plays a role only in the first part. The second part is based on the fact (first noticed by Stembridge [14]) that the $q, t$-Kostka polynomials $K_{\lambda \mu}(q, t)$ for $\mu$ a two-column partition may be given a completely explicit expression for all $\lambda$.

Our point of departure here is the classical identity

$$
\partial_{p_{1}} \tilde{H}_{\mu}(x ; q, t)=\sum_{\nu \rightarrow \mu} c_{\mu \nu}(q, t) \tilde{H}_{\nu}(x ; q, t)
$$


In a form given in the "Lattice diagram" paper [3]. To this end we need to recall some of the notation and definitions used there. For a partition $\mu$ with $m$ corners, denote by

$$
\nu^{(1)}, \nu^{(2)}, \ldots, \nu^{(m)}
$$

the partitions obtained by removing one of the corners of $\mu$ in succession from left to right. Let us call these corners $A_{1}, A_{2}, \ldots, A_{m}$. That is

$$
A_{1}=\mu / \nu^{(1)}, A_{2}=\mu / \nu^{(2)}, \ldots, A_{m}=\mu / \nu^{(d)}
$$

The weights of these corners are respectively denoted

$$
x_{1}, x_{2}, \ldots, x_{d}
$$

More precisely, if the $i^{\text {th }}$ corner, has coleg $l_{i}^{\prime}$ and coarm $a_{i}^{\prime}$ in $\mu$, we set

$$
x_{i}=x_{i}=t^{l_{i}^{\prime}} q^{a_{i}^{\prime}}
$$

Moreover, we call $B_{i}$ the cell of $\mu$ that lies below $A_{i}$ at the intersection of the column of $A_{i}$ with the row of $A_{i+1}$, and call $u_{i}$ its weight. We referred to the $B_{i}^{\prime} s$ the "inner corners" of $\mu$. Clearly we must have

$$
u_{i}=t^{l_{i+1}^{\prime}} q^{a_{i}^{\prime}} \quad(\text { for } \quad i=1,2, \ldots, m-1)
$$

The picture is completed by adding three more cells $A_{0}$ and $B_{0}, B_{m}$ with weights

$$
u_{0}=t^{l_{1}^{\prime}} / q, u_{m}=q^{a_{m}^{\prime}} / t \quad \text { and } \quad x_{0}=1 / t q .
$$

To appreciate the geometric significance of these weights, in the figure below we illustrate a 4-corner Ferrers diagram with corner cells $A_{0}, A_{1}, A_{2}, A_{3}, A_{4}$ and inner corner cells $B_{0}, B_{1}, B_{2}, B_{3}, B_{4}$.

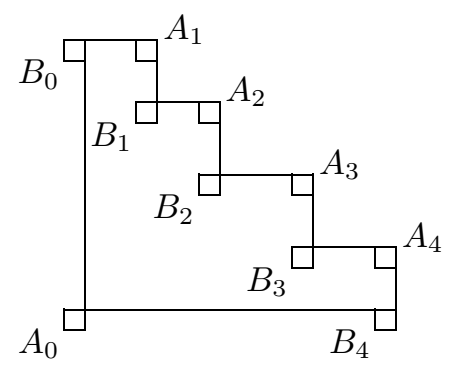

It was shown in [1] that the original dual Pieri coefficients $c_{\mu \nu}(q, t)$ undergo massive cancellations which reduce them to relatively simpler expressions in terms of the corner weights. This results in the formula

$$
c_{\mu \nu^{(i)}}(q, t)=\frac{1}{(1-1 / t)(1-1 / q)} \frac{1}{x_{i}} \frac{\prod_{j=0}^{m}\left(x_{i}-u_{j}\right)}{\prod_{j=1 ; j \neq i}^{m}\left(x_{i}-x_{j}\right)}
$$


In the two column case $\mu=2^{b} 1^{a-b}$ the diagram degenerates to

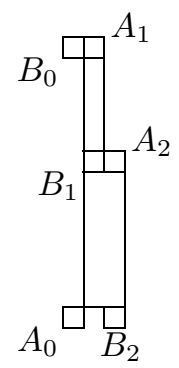

with $A_{0}=(-1,-1), A_{1}=(1, a), A_{2}=(2, b)$ and $B_{0}=(-1, a), B_{1}=(1, b), B_{2}=(2,-1)$. To use 3.1 with the $c_{\mu \nu}(q, t)$ given by 3.5 , it will be convenient to set

$$
\tilde{H}_{2^{b} 1^{a-b}}(x ; t)=\tilde{H}_{a, b}, \quad \tilde{H}_{2^{b} 1^{a-b-1}}(x ; t)=\tilde{H}_{a-1, b}, \quad \tilde{H}_{2^{b-1} 1^{a-b+1}}(x ; t)=\tilde{H}_{a, b-1},
$$

and rewrite 3.1 as

$$
\partial_{p_{1}} \tilde{H}_{a, b}=c_{a}(q, t) \tilde{H}_{a-1, b}+c_{b}(q, t) \tilde{H}_{a, b-1} .
$$

with 3.5 giving

$$
c_{a}(q, t)=\frac{1}{(1-1 / t)(1-1 / q)} \frac{1}{x_{1}} \frac{\left(x_{1}-u_{0}\right)\left(x_{1}-u_{1}\right)\left(x_{1}-u_{2}\right)}{\left(x_{1}-x_{2}\right)}
$$

and

$$
c_{b}(q, t)=\frac{1}{(1-1 / t)(1-1 / q)} \frac{1}{x_{2}} \frac{\left(x_{2}-u_{0}\right)\left(x_{2}-u_{1}\right)\left(x_{2}-u_{2}\right)}{\left(x_{2}-x_{1}\right)}
$$

Since in this case we have

$$
x_{0}=1 / t q, \quad x_{1}=t^{a-1}, \quad x_{2}=t^{b-1} q
$$

and

$$
u_{0}=t^{a-1} / q, \quad u_{1}=t^{b-1}, \quad u_{2}=q / t,
$$

formulas 3.7 and 3.8 give

$$
c_{a}(q, t)=\frac{1}{(1-1 / t)(1-1 / q)} \frac{1}{t^{a-1}} \frac{\left(t^{a-1}-t^{a-1} / q\right)\left(t^{a-1}-t^{b-1}\right)\left(t^{a-1}-q / t\right)}{\left(t^{a-1}-t^{b-1} q\right)}
$$

and

$$
c_{b}(q, t)=\frac{1}{(1-1 / t)(1-1 / q)} \frac{1}{t^{b-1} q} \frac{\left(t^{b-1} q-t^{a-1} / q\right)\left(t^{b-1} q-t^{b-1}\right)\left(t^{b-1} q-q / t\right)}{\left(t^{b-1} q-t^{a-1}\right)}
$$

Canceling repeated factors finally yields

$$
c_{a}(q, t)=\frac{\left(t^{a-b}-1\right)\left(t^{a}-q\right)}{(t-1)\left(t^{a-b}-q\right)}
$$

and

$$
c_{b}(q, t)=\frac{\left(t^{b}-1\right)\left(t^{a-b}-q^{2}\right)}{(t-1)\left(t^{a-b}-q\right)}
$$


and 3.6 becomes

$$
\partial_{p_{1}} \tilde{H}_{a, b}=\frac{\left(t^{a-b}-1\right)\left(t^{a}-q\right)}{(t-1)\left(t^{a-b}-q\right)} \tilde{H}_{a-1, b}+\frac{\left(t^{b}-1\right)\left(t^{a-b}-q^{2}\right)}{(t-1)\left(t^{a-b}-q\right)} \tilde{H}_{a, b-1} .
$$

Now Science Fiction suggests that the rational functions appearing in the right hand side can be unraveled by means of the substitutions

$$
\tilde{H}_{a-1, b}=\phi_{a, b}+T_{a-1, b} \psi_{a, b}, \quad \tilde{H}_{a, b-1}=\phi_{a, b}+T_{a, b-1} \psi_{a, b}
$$

with

$$
T_{a-1, b}=t^{\left(\begin{array}{c}
a-1 \\
2
\end{array}\right)+\left(\begin{array}{c}
b \\
2
\end{array}\right)} q^{b}, \quad T_{a, b-1}=t^{\left(\begin{array}{c}
a \\
2
\end{array}\right)+\left(\begin{array}{c}
b-1 \\
2
\end{array}\right)} q^{b} .
$$

In fact, using 3.11 in 3.10 gives

\section{Proposition 3.1}

$$
\partial_{p_{1}} \tilde{H}_{a, b}=(1+q)[b]_{t} \tilde{H}_{a, b-1}+[a-b]_{t}\left(t^{b} \phi_{a, b}+T_{a-1, b} \psi_{a, b}\right)
$$

Proof

To begin note that from 3.11 we derive

$$
\phi_{a, b}=\frac{T_{a, b-1} \tilde{H}_{a-1, b}-T_{a-1, b} \tilde{H}_{a, b-1}}{T_{a, b-1}-T_{a-1, b}}, \quad \psi_{a, b}=\frac{\tilde{H}_{a, b-1}-\tilde{H}_{a-1, b}}{T_{a, b-1}-T_{a-1, b}}
$$

Now from the definitions in 3.12 it follows that

$$
T_{a, b-1} / T_{a-1, b}=t^{a-b} / q
$$

and using this in 3.14 gives

$$
\text { a) } \phi_{a, b}=\frac{t^{a-b} \tilde{H}_{a-1, b}-q \tilde{H}_{a, b-1}}{t^{a-b}-q}, \quad \text { b) } \quad T_{a-1, b} \psi_{a, b}=q \frac{\tilde{H}_{a, b-1}-\tilde{H}_{a-1, b}}{t^{a-b}-q}
$$

Using these expresions in 3.13 yields

$$
\begin{aligned}
\partial_{p_{1}} \tilde{H}_{a, b} & =(1+q)[b]_{t} \tilde{H}_{a, b-1}+[a-b]_{t}\left(\frac{t^{a} \tilde{H}_{a-1, b}-t^{b} q \tilde{H}_{a, b-1}}{t^{a-b}-q}+\frac{q \tilde{H}_{a, b-1}-q \tilde{H}_{a-1, b}}{t^{a-b}-q}\right) \\
& =\frac{(1+q)\left(1-t^{b}\right)\left(t^{a-b}-q\right) \tilde{H}_{a, b-1}+\left(1-t^{a-b}\right)\left(\left(t^{a}-q\right) \tilde{H}_{a-1, b}+q\left(1-t^{b}\right) \tilde{H}_{a, b-1}\right)}{(1-t)\left(t^{a-b}-q\right)} \\
& =\frac{\left(1-t^{b}\right)\left((1+q)\left(t^{a-b}-q\right)+q\left(1-t^{a-b}\right)\right) \tilde{H}_{a, b-1}}{(1-t)\left(t^{a-b}-q\right)}+\frac{\left(1-t^{a-b}\right)\left(t^{a}-q\right) \tilde{H}_{a-1, b}}{(1-t)\left(t^{a-b}-q\right)}
\end{aligned}
$$

and we see that 3.13 is simply another way of writing 3.10 , since

$$
(1+q)\left(t^{a-b}-q\right)+q\left(1-t^{a-b}\right)=t^{a-b}-q+q t^{a-b}-q^{2}+q-q t^{a-b}=t^{a-b}-q^{2}
$$

(*) Here as customary $[x]_{t}=\left(1-t^{x}\right) /(1-t)$ 
This proposition has the following immediate corollary

\section{Proposition 3.2}

The recursion in 1.7 is equivalent to the identity

$$
t^{b} \phi_{a, b}+T_{a-1, b} \psi_{a, b}=t^{b} \tilde{H}_{2^{b}, 1^{a-b-1}}(q / t, t)
$$

\section{Proof}

Note that converting th first two members of I.7 to the present notation we get

$$
\partial_{p_{1}} \tilde{H}_{a, b}=(1+q)[b]_{t} \tilde{H}_{a, b-1}+[a-b]_{t} t^{b} \tilde{H}_{2^{b}, 1^{a-b-1}}(q / t, t)
$$

Comparing this with 3.13 we see that I.7 is equivalent to

$$
[a-b]_{t}\left(t^{b} \phi_{a, b}+T_{a-1, b} \psi_{a, b}\right)=[a-b]_{t} t^{b} \tilde{H}_{2^{b}, 1^{a-b-1}}(q / t, t)
$$

and 3.17 is simply obtained by cancelling the common factor.

\section{Enter Hall-Littlewood polynomials}

Our point of departure is the Macdonald specialization

$$
\tilde{H}_{\mu}[1-x ; q, t]=\prod_{i=1}^{l(\mu)} \prod_{j=1}^{\mu_{i}}\left(1-x t^{i-1} q^{j-1}\right)
$$

Now, for $\mu=2^{b} 1^{a-b}, 4.1$ gives

$$
\tilde{H}_{2^{b} 1^{a-b}}[1-x ; q, t]=\prod_{i=1}^{a}\left(1-x t^{i-1}\right) \times \prod_{i=1}^{b}\left(1-q x t^{i-1}\right)=(x ; t)_{a}(q x ; t)_{b} .
$$

Recalling that we have set $\tilde{H}_{a, b}(X ; q, t)=\tilde{H}_{2^{b} 1^{a-b}}(X ; q, t)$ it will be convenient to also set

$$
\tilde{H}_{a, b}(X ; t)=\tilde{H}_{2^{b} 1^{a-b}}(X ; 0, t)
$$

and 4.2 reduces to

$$
\tilde{H}_{a, b}(1-x ; t)=(x ; t)_{a}
$$

Since the Macdonald polynomials are triangularly related to the Hall-Littlewood polynomials we will have coefficients $\theta_{s}^{a, b}(q, t)$ such that

$$
\tilde{H}_{a, b}(X ; q, t)=\sum_{s=0}^{b} \tilde{H}_{a+s, b-s}(X ; t) \theta_{s}^{a, b}(q, t)
$$

specialising to the alphabet $X=1-x$ and using 4.2 and 4.3 this relation becomes

$$
(x ; t)_{a}(q x ; t)_{b}=\sum_{s=0}^{b}(x ; t)_{a+s} \theta_{s}^{a, b}(q, t)
$$


From which we derive that

$$
\theta_{s}^{a, b}(q, t)=\left.(x ; t)_{a}(q x ; t)_{b}\right|_{(x ; t)_{a+s}}
$$

and 4.4 becomes

$$
\tilde{H}_{a, b}(X ; q, t)=\left.\sum_{s=0}^{b} \tilde{H}_{a+s, b-s}(X ; t)(x ; t)_{a}(q x ; t)_{b}\right|_{(x ; t)_{a+s}}
$$

To complete our proof of the recursion it will be more convenient to work with our parameters $a, b$ shifted to $a+1, b$, and work with the symmetric functions in 3.16 replaced by

$$
\text { a) } \phi_{a+1, b}=\frac{t^{a-b+1} \tilde{H}_{a, b}-q \tilde{H}_{a+1, b-1}}{t^{a-b+1}-q}, \quad \text { b) } T_{a, b} \psi_{a+1, b}=-q \frac{\tilde{H}_{a, b}-\tilde{H}_{a+1, b-1}}{t^{a-b+1}-q}
$$

Our goal here will then be to prove the identity

$$
t^{b} \phi_{a+1, b}+T_{a, b} \psi_{a+1, b}=t^{b} \tilde{H}_{a, b}(q / t, t)
$$

With the same parameter changes the definitions in 3.10 give

$$
\tilde{H}_{a, b}(X ; q, t)=\phi_{a+1, b}+T_{a, b} \psi_{a+1, b}
$$

Now making the replacements $a \rightarrow a+1$ and $b \rightarrow b-1$ in 4.7 gives

$$
\begin{aligned}
\tilde{H}_{a+1, b-1}(X ; q, t) & =\left.\sum_{s=0}^{b-1} \tilde{H}_{a+1+s, b-s-1}(X ; t)(x ; t)_{a+1}(q x ; t)_{b-1}\right|_{(x ; t)_{a+1+s}} \\
& =\left.\sum_{s=1}^{b} \tilde{H}_{a+s, b-s}(X ; t)(x ; t)_{a+1}(q x ; t)_{b-1}\right|_{(x ; t)_{a+s}} .
\end{aligned}
$$

Using this and 4.7 in 4.8 a) we get

$$
\begin{aligned}
\left(t^{a-b+1}-q\right) \phi_{a+1, b}= & \left.t^{a-b+1} \tilde{H}_{a, b}(X ; t)(x ; t)_{a}(q x ; t)_{b}\right|_{(x ; t)_{a}} \\
& +\left.\sum_{s=1}^{b} \tilde{H}_{a+s, b-s}(X ; t)\left(t^{a-b+1}(x ; t)_{a}(q x ; t)_{b}-q(x ; t)_{a+1}(q x ; t)_{b-1}\right)\right|_{(x ; t)_{a+s}}
\end{aligned}
$$

Now we have

$$
\begin{aligned}
t^{a-b+1}(x ; t)_{a}(q x ; t)_{b}-q(x ; t)_{a+1}(q x ; t)_{b-1} & =(x ; t)_{a}(q x ; t)_{b-1}\left(t^{a-b+1}\left(1-x q t^{b-1}\right)-q\left(1-x t^{a}\right)\right) \\
& =\left(t^{a-b+1}-q\right)(x ; t)_{a}(q x ; t)_{b-1}
\end{aligned}
$$

and 4.12 becomes

$$
\phi_{a+1, b}=\left.\frac{t^{a-b+1}}{t^{a-b+1}-q} \tilde{H}_{a, b}(X ; t)(x ; t)_{a}(q x ; t)_{b}\right|_{(x ; t)_{a}}+\left.\sum_{s=1}^{b} \tilde{H}_{a+s, b-s}(X ; t)(x ; t)_{a}(q x ; t)_{b-1}\right|_{(x ; t)_{a+s}}
$$


To get $T_{a, b} \psi_{a+1, b}$ we note that

$$
\begin{aligned}
\tilde{H}_{a, b}(X ; q, t)-\tilde{H}_{a+1, b-1}(X ; q, t) & =\left.\sum_{s=0}^{b} \tilde{H}_{a+s, b-s}(X ; t)(x ; t)_{a}(q x ; t)_{b}\right|_{(x ; t)_{a+s}} \\
& -\left.\sum_{s=1}^{b} \tilde{H}_{a+s, b-s}(X ; t)(x ; t)_{a+1}(q x ; t)_{b-1}\right|_{(x ; t)_{a+s}} \\
& =\left.\tilde{H}_{a, b}(X ; t)(x ; t)_{a}(q x ; t)_{b}\right|_{(x ; t)_{a}}+\sum_{s=1}^{b} \tilde{H}_{a+s, b-s}(X ; t)(x ; t)_{a}(q x ; t)_{b-1}\left(1-q x t^{b-1}-1+x t^{a}\right) \\
& =\left.\tilde{H}_{a, b}(X ; t)(x ; t)_{a}(q x ; t)_{b}\right|_{(x ; t)_{a}}+x t^{b-1} \sum_{s=1}^{b} \tilde{H}_{a+s, b-s}(X ; t)(x ; t)_{a}(q x ; t)_{b-1}\left(-q+t^{a-b+1}\right)
\end{aligned}
$$

and $4.8 \mathrm{~b}$ ) becomes

$$
T_{a, b} \psi_{a+1, b}=\left.\left.\frac{-q}{t^{a-b+1}-q} \tilde{H}_{a, b}(X ; t)(x ; t)_{a}(q x ; t)_{b}\right|_{(x ; t)_{a}} q x t^{b-1} \sum_{s=1}^{b} \tilde{H}_{a+s, b-s}(X ; t)(x ; t)_{a}(q x ; t)_{b-1}\right|_{(x ; t)_{a+s}}
$$

Next note that 4.7 gives

$$
t^{b} \tilde{H}_{a, b}(X ; q / t, t)=\left.t^{b} \sum_{s=0}^{b} \tilde{H}_{a+s, b-s}(X ; t)(x ; t)_{a}(q x / t ; t)_{b}\right|_{(x ; t)_{a+s}}
$$

and since

$$
t^{b}(q x / t ; t)_{b}=t^{b}(1-q x / t)(1-q x) \cdots\left(1-q x t^{b-2}\right)=t^{b-1}(t-q x)(q x ; t)_{b-1}
$$

4.16 becomes

$$
t^{b} \tilde{H}_{a, b}(X ; q / t, t)=\left.\left(t^{b}-t^{b-1} q x\right) \sum_{s=0}^{b} \tilde{H}_{a+s, b-s}(X ; t)(x ; t)_{a}(q x ; t)_{b-1}\right|_{(x ; t)_{a+s}}
$$

Thus to prove 4.9 we need only show that the right hand side of 4.16 can also be obtained by adding the right hand sides of the following two equalities.

$$
\begin{aligned}
& t^{b} \phi_{a, b}=\left.\frac{t^{a+1}}{t^{a-b+1}-q} \tilde{H}_{a, b}(X ; t)(x ; t)_{a}(q x ; t)_{b}\right|_{(x ; t)_{a}}+\left.t^{b} \sum_{s=1}^{b} \tilde{H}_{a+s, b-s}(X ; t)(x ; t)_{a}(q x ; t)_{b-1}\right|_{(x ; t)_{a+s}} \\
& \psi_{a, b}=\left.\frac{-q}{t^{a-b+1}-q} \tilde{H}_{a, b}(X ; t)(x ; t)_{a}(q x ; t)_{b}\right|_{(x ; t)_{a}}-\left.q x t^{b-1} \sum_{s=1}^{b} \tilde{H}_{a+s, b-s}(X ; t)(x ; t)_{a}(q x ; t)_{b-1}\right|_{(x ; t)_{a+s}}
\end{aligned}
$$

In fact doing this gives this gives

$$
\begin{aligned}
t^{b} \phi_{a, b}+\psi_{a, b}=\frac{t^{a+1}-q}{t^{a-b+1}-q} & \left.\tilde{H}_{a, b}(X ; t)(x ; t)_{a}(q x ; t)_{b}\right|_{(x ; t)_{a}} \\
& +\left.\left(t^{b}-q x t^{b-1}\right) \sum_{s=1}^{b} \tilde{H}_{a+s, b-s}(X ; t)(x ; t)_{a}(q x ; t)_{b-1}\right|_{(x ; t)_{a+s}}
\end{aligned}
$$


Comparing with the right hand side of 4.17 reduces us to proving the equality

$$
\left.\frac{t^{a+1}-q}{t^{a-b+1}-q}(x ; t)_{a}(q x ; t)_{b}\right|_{(x ; t)_{a}}=\left.\left(t^{b}-q x t^{b-1}\right)(x ; t)_{a}(q x ; t)_{b-1}\right|_{(x ; t)_{a}}
$$

0r, equivalently show that

$$
\left(t^{a+1}-q\right)(x ; t)_{a}(q x ; t)_{b}-\left.\left(t^{a-b+1}-q\right)\left(t^{b}-q x t^{b-1}\right)(x ; t)_{a}(q x ; t)_{b-1}\right|_{(x ; t)_{a}}=0
$$

Now this is best rewritten in the form

$$
\left.\left(\left(t^{a+1}-q\right)\left(1-q x t^{b-1}\right)-\left(t^{a-b+1}-q\right)\left(t^{b}-q x t^{b-1}\right)\right)(x ; t)_{a}(q x ; t)_{b-1}\right|_{(x ; t)_{a}}=0
$$

But a simple calculation gives

$$
\left(t^{a+1}-q\right)\left(1-q x t^{b-1}\right)-\left(t^{a-b+1}-q\right)\left(t^{b}-q x t^{b-1}\right)=q\left(t^{b}-1\right)\left(1-x t^{a}\right)
$$

and 4.19 becomes

$$
\left.q\left(t^{b}-1\right)\left(1-x t^{a}\right)(x ; t)_{a}(q x ; t)_{b-1}\right|_{(x ; t)_{a}}=0
$$

or better

$$
\left.q\left(t^{b}-1\right)(x ; t)_{a+1}(q x ; t)_{b-1}\right|_{(x ; t)_{a}}=0
$$

which is patently obvious.

This completes the proof of 4.9 and thus by Proposition 3.2 the identity in I.7 as well as the recursion in I.6 are thereby established. 


\section{The $[3,2,1]$ case.}

For convenience we will set

$$
\alpha=[3,2]=\text { 田, } \beta=[3,1,1]=\text { 目, } \gamma=[2,2,1]=\text { 早 } .
$$

and also use the same symbols to represent the corresponding modules as well as their Frobenius characteristics. Science Fiction states that in the this case the $S_{n}$ module

\section{田、助、田}

decomposes into the direct sum of $\mathbf{7}$ modules precisely as the union of three sets decomposes into $\mathbf{7}$ disjoint subsets. Thus this decomposition may be depicted by a 3-subset Venn Diagram as follows.

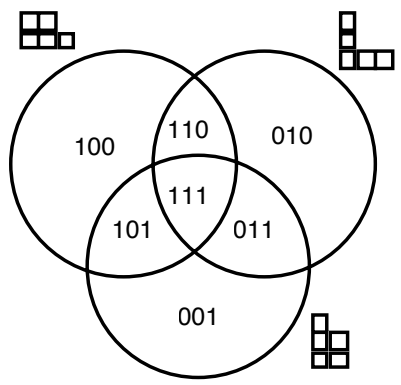

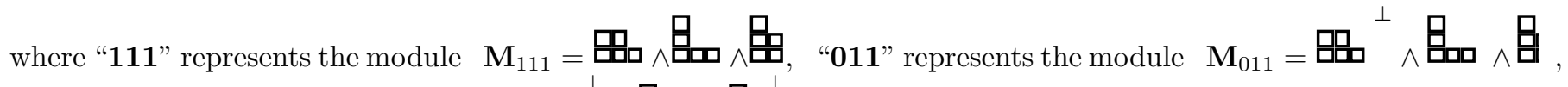

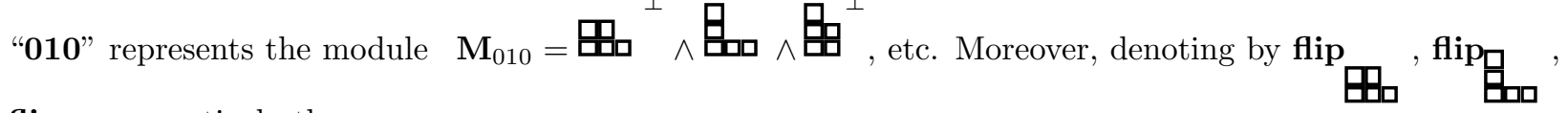
${ }^{\text {flip }}$ 盟 respectively the maps

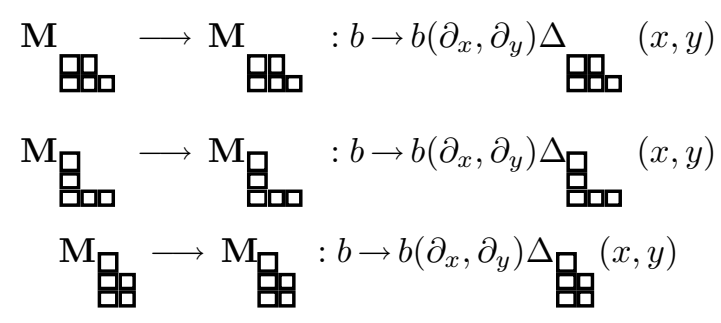

Science Fiction asserts that
a) $\operatorname{flip}_{111} \mathbf{M}_{100}$
b) flip $\mathbf{M}_{110}=\mathbf{M}_{101}$,
a) $\mathrm{flip}_{\text {日 }} \mathbf{M}_{111}=\mathbf{M}_{010}$ ，
b) ${ }^{\text {flip }}{ }_{\text {D }} \mathbf{M}_{011}=\mathbf{M}_{110}$,
a) flip $_{\mathrm{H}_{111}} \mathbf{M}_{110} \mathbf{M}_{001}$, 由
b) flip $_{\text {要 }} \mathbf{M}_{101}=\mathbf{M}_{011}$ 
Denoting by $\phi_{111}, \phi_{011}, \phi_{101}, \phi_{110}, \phi_{100}, \phi_{010}, \phi_{001}$, the corresponding Frobenius characteristics we see that these equations respoectively yield the following identities
a) $\phi_{100}=T_{\alpha} \downarrow \phi_{111}$,
b) $\phi_{101}=T_{\alpha} \downarrow \phi_{110}$,
a) $\phi_{010}=T_{\beta} \downarrow \phi_{111}$,
b) $\phi_{110}=T_{\beta} \downarrow \phi_{011}$,
a) $\phi_{010}=T_{\gamma} \downarrow \phi_{111}$,
b) $\phi_{011}=T_{\gamma} \downarrow \phi_{101}$,

Note then that from $5.1^{*} \mathrm{~b}$ ) and $5.2^{*} \mathrm{~b}$ ) we derive that

$$
\phi_{101}=T_{\alpha} \downarrow\left(T_{\beta} \downarrow \phi_{011}\right)=\frac{T \alpha}{T_{\beta}} \phi_{011}
$$

or better

$$
T_{\alpha} \phi_{011}=T_{\beta} \phi_{101}
$$

similarly we derive that

$$
T_{\beta} \phi_{101}=T_{\gamma} \phi_{110}
$$

Denoting by $\phi_{3}$ the common value we get that

$$
\text { a) } \phi_{011}=\frac{\phi_{2}}{T_{\alpha}} \quad \text { b) } \phi_{101}=\frac{\phi_{2}}{T_{\beta}} \quad \text { c) } \quad \phi_{110}=\frac{\phi_{2}}{T_{\gamma}}
$$

In a similar manner from $5.1^{*}$ a), $5.2^{*}$ a), $5.3^{*}$ a), we derive that setting

$$
\phi_{1}=T_{\alpha} T_{\beta} T_{\gamma} \downarrow \phi_{111}
$$

we get
a) $\quad \phi_{100}=\frac{\phi_{2}}{T_{\beta} T_{\gamma}}$
b) $\phi_{010}=\frac{\phi_{2}}{T_{\alpha} T_{\gamma}}$
c) $\phi_{001}=\frac{\phi_{2}}{T_{\alpha} T_{\beta}}$

In particular combining these identities with the direct sum decompositions expressed by the Venn diagram at the end of the paper, and setting $\phi_{3}=\phi_{111}$ we derive that

$$
\begin{gathered}
\tilde{H}_{\text {田 }}=\phi_{111}+\phi_{101}+\phi_{110}+\phi_{100}=\phi_{3}+\frac{\phi_{2}}{T_{\beta}}+\frac{\phi_{2}}{T_{\gamma}}+\frac{\phi_{1}}{T_{\beta} T_{\gamma}} \\
\tilde{H}_{\text {国 }}=\phi_{111}+\phi_{011}+\phi_{110}+\phi_{010}=\phi_{3}+\frac{\phi_{2}}{T_{\alpha}}+\frac{\phi_{2}}{T_{\gamma}}+\frac{\phi_{1}}{T_{\alpha} T_{\gamma}} \\
\tilde{H}_{\text {田 }}=\phi_{111}+\phi_{011}+\phi_{101}+\phi_{001}=\phi_{3}+\frac{\phi_{2}}{T_{\alpha}}+\frac{\phi_{2}}{T_{\beta}}+\frac{\phi_{1}}{T_{\alpha} T_{\beta}}
\end{gathered}
$$

Our goal in this section is to show how the restriction to $\mathcal{S}_{5}$ of the Garsia-Haiman module $\mathbf{M}_{321}$ may be decomposed as a direct sum of submodules isomorphic to the submodules appearing in our Venn diagram. From this decomposition it will ultimately follow that

$$
\partial_{p_{1}} \tilde{H}_{321}=\left((1+t)(1+q)+t^{2}+q^{2}\right) \phi_{3}+(1+t)(1+q)\left(t^{2}+t q+q^{2}\right) \frac{\phi_{2}}{t^{4} q^{4}}+\left(t q(1+t)(1+q)+t^{2}+q^{2}\right) \frac{\phi_{1}}{t^{7} q^{7}} 5.10
$$


Our final task will be to reassemble the resulting terms into what we might believe is a 321-analogue of the two decompositions in 5.6 and 5.7, namely

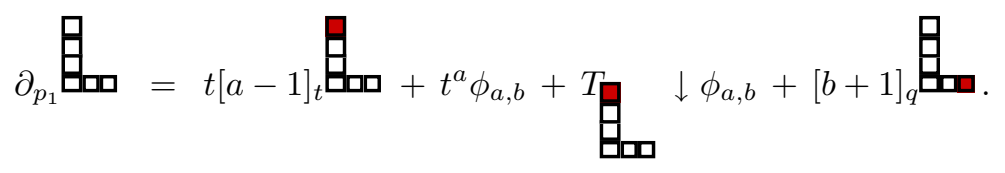

and

$$
\partial_{p_{1}} \text { 苖 }=t^{b}[a-b]_{t} \phi_{a, b}+[a-b]_{t} T_{\text {男 }} \downarrow \phi_{a, b}+(1+q)[b]_{t} \text { 畀。 }
$$

Note that setting

$$
T_{\alpha}=T_{\text {田 }}=t^{2} q^{4} \quad, T_{\beta}=T_{\text {且 }}=t^{3} q^{3} \quad, T_{\gamma}=T_{\text {田 }}=t^{4} q^{2},
$$

the relations in 5.7, 5.8 and 5.9 become

$$
\begin{gathered}
\tilde{H}_{\text {田 }}=\phi_{111}+\phi_{101}+\phi_{110}+\phi_{100}=\phi_{3}+\frac{\phi_{2}}{t^{3} q^{3}}+\frac{\phi_{2}}{t^{4} q^{2}}+\frac{\phi_{1}}{t^{7} q^{5}} \\
\tilde{H}_{\text {目 }}=\phi_{111}+\phi_{011}+\phi_{110}+\phi_{010}=\phi_{3}+\frac{\phi_{2}}{t^{2} q^{4}}+\frac{\phi_{2}}{t^{4} q^{2}}+\frac{\phi_{1}}{t^{6} q^{6}} \\
\tilde{H}_{\text {思 }}=\phi_{111}+\phi_{011}+\phi_{101}+\phi_{001}=\phi_{3}+\frac{\phi_{2}}{t^{2} q^{4}}+\frac{\phi_{2}}{t^{3} q^{3}}+\frac{\phi_{1}}{t^{5} q^{7}}
\end{gathered}
$$

This given a possible 321 -analogue of 5.11 and 5.12 could be

$$
\partial_{p_{1}} \tilde{H}_{321}=(1+t)(1+q) \tilde{H}_{\boldsymbol{H}_{\mathbf{0}}}+\left(q^{2}+t^{2}\right) \phi_{111}+(1+t)(1+q) \phi_{101}+\phi_{100}+\phi_{001}
$$

since this is one of the ways to use the identities in $5.7^{*}-5.9^{*}$ to re-express 5.10 making maximal use of one of the $\tilde{H}_{\mu}$ for $\mu \in\{[3,2],[3,1,1],[2,2,1]\}$. We can easily check that no more than three copies of $\tilde{H}_{32}$ or $\tilde{H}_{221}$ can be sutracted from $\partial_{p_{1}} \tilde{H}_{321}$ and still yielding a positive remainder. More precisely $\partial_{p_{1}} \tilde{H}_{321}-\left(1+t+t^{2}\right) \tilde{H}_{32}$ and $\partial_{p_{1}} \tilde{H}_{321}-\left(1+q+q^{2}\right) \tilde{H}_{221}$ are both Schur positive, but any more subtractions produces negatives.

Our next task is to derive the identity in 5.10. We will proceed first by the "kicking" method combined with a minimal use of Science Fiction as we did for the two-column case. This done we will illustrate a general algorithm for obtaining such an identity for any partiton by applying the algorithm to the 321-case.

Our point of departure are the two kicking identities

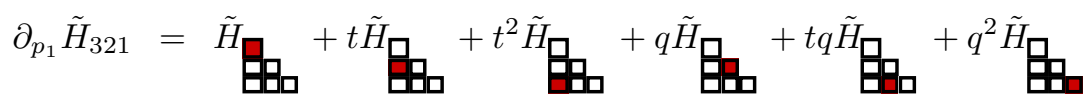




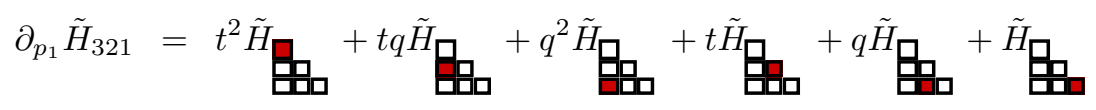

Using the equivalences

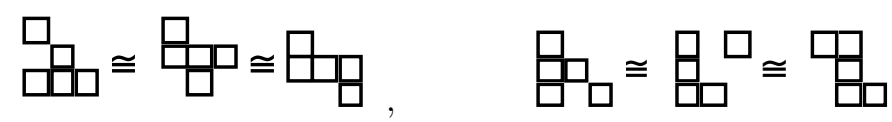

The identities in 1.14 and 5.15 become

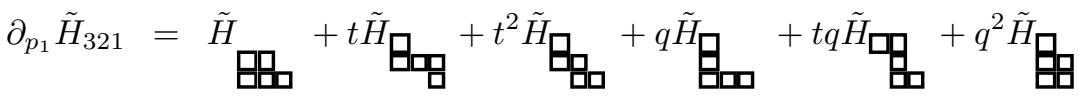

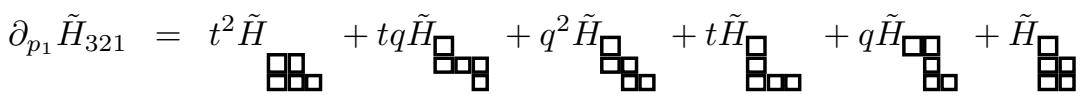

and by subtraction we derive that

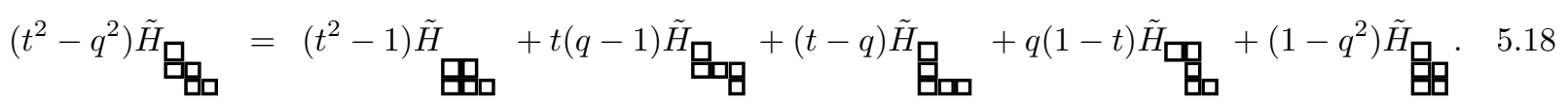

But we cannot derive anything from this until we construct $\tilde{H}_{\mathbf{Z}}$ and $\tilde{H}_{\mathbf{Z}}$. To do that we carry out another kicking scheme as decribed by the following display

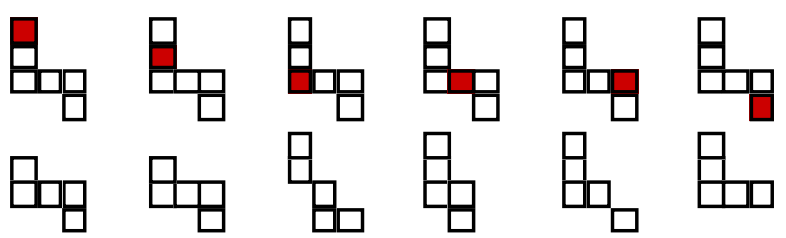

and derive the two identities

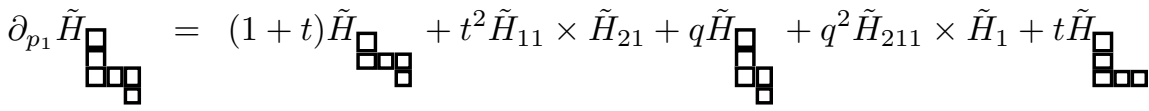

$$
\begin{aligned}
& \partial_{p_{1}} \tilde{H}_{\mathrm{g}}=\left(t^{2}+t\right) \tilde{H}_{\mathrm{Q}}+q^{2} \tilde{H}_{11} \times \tilde{H}_{21}+q \tilde{H}_{\text {㚣 }}+t \tilde{H}_{211} \times \tilde{H}_{1}+\tilde{H}_{\text {日 }}
\end{aligned}
$$

and by subtraction we derive (note $\tilde{H}_{\mathrm{B}}$ cancels!)

$$
\left(t^{2}-1\right) \tilde{H}_{\mathbf{Z}}=\left(t^{2}-q^{2}\right) \tilde{H}_{11} \times \tilde{H}_{21}+q^{2} \tilde{H}_{211} \times \tilde{H}_{1}+t \tilde{H}_{\mathbf{⿴}}
$$

Using the Pieri rules

$$
\tilde{H}_{11} \times \tilde{H}_{21}=\frac{(q-1)\left(q^{2}-t\right)}{\left(q-t^{2}\right)\left(q^{2}-t^{3}\right)} \tilde{H}_{2111}+\frac{\left(1-t^{2}\right)(q-1)\left(q^{2}-t\right)}{(q-t)^{2}(q+t)\left(q-t^{2}\right)} \tilde{H}_{221} \frac{\left(1-t^{2}\right)(q-1)\left(q-t^{2}\right)}{(q-t)^{2}\left(q^{2}-t^{3}\right)} \tilde{H}_{311}+\frac{(1+t)(t-1)^{2}}{(q+t)(q-t)^{2}} \tilde{H}_{32}
$$




$$
\tilde{H}_{211} \times \tilde{H}_{1}=\frac{(q-1)\left(q^{2}-t\right)}{\left(q-t^{2}\right)\left(q^{2}-t^{3}\right)} \tilde{H}_{2111}+\frac{\left(t^{2}-1\right)(1-q)}{(q-t)\left(q-t^{2}\right)} \tilde{H}_{221}+\frac{(1-t)\left(q-t^{3}\right)}{(q-t)\left(q^{2}-t^{3}\right)} \tilde{H}_{311}
$$

The equality in 5.21 yields

$$
{ }^{{ }^{H}} \mathbf{Q}_{\mathbf{Z}}=\frac{q-1}{q-t} \tilde{H}_{\mathbf{⿴}}+\frac{1-t}{q-t} \tilde{H} \mathbf{⿴}
$$

and by conjugation we obtain

$$
\tilde{H}_{\text {思 }}=\frac{t-1}{t-q} \tilde{H}_{\mathbf{m}}+\frac{1-q}{t-q} \tilde{H}_{\text {田 }}
$$

Using 5.22 and 5.23 in 5.18 gives, after simplifications

$$
\tilde{H}_{\mathbf{Z}_{\mathbf{0}}}=\frac{(q-1)\left(q^{2}-t\right)}{(q+t)(q-t)^{2}} \tilde{H}_{221}+\frac{(1-t)(q-1)}{(q-t)^{2}} \tilde{H}_{311}+\frac{(1-t)\left(q-t^{2}\right)}{(q+t)(q-t)^{2}} \tilde{H}_{32}
$$

Notwithstanding the rationality of these expressions, substituting in 5.22, 5.23 and 5.24 the relations in $5.7^{*}$, $5.9^{*}$ and $5.9^{*}$, gives

$$
\begin{aligned}
& \tilde{H}_{\mathrm{Z}_{\mathrm{Z}}}=f_{3}+\frac{\phi_{2}}{t^{4} q^{2}}+\frac{\phi_{2}}{t^{3} q^{4}}+\frac{\phi_{1}}{t^{7} q^{6}} \\
& \tilde{H}_{\text {甼 }}=f_{3}+\frac{\phi_{2}}{t^{2} q^{4}}+\frac{\phi_{2}}{t^{4} q^{3}}+\frac{\phi_{1}}{t^{6} q^{7}} \\
& { }^{\tilde{H}_{\text {女 }}} \mathrm{B}_{\mathrm{Z}}=f_{3}+\frac{\phi_{2}}{t^{4} q^{3}}+\frac{\phi_{2}}{t^{3} q^{4}}+\frac{\phi_{1}}{t^{7} q^{7}}
\end{aligned}
$$

We are now finally in a position to prove 5.10 . To do this we use these last relations and $5.7^{*}, 5.9^{*}$ and $5.9^{*}$ in 5.17 and obtain

$$
\begin{gathered}
\partial_{p_{1}} \tilde{H}_{321}=t^{2}\left(\phi_{3}+\frac{\phi_{2}}{t^{3} q^{3}}+\frac{\phi_{2}}{t^{4} q^{2}}+\frac{\phi_{1}}{t^{7} q^{5}}\right)+t q\left(f_{3}+\frac{\phi_{2}}{t^{4} q^{2}}+\frac{\phi_{2}}{t^{3} q^{4}}+\frac{\phi_{1}}{t^{7} q^{6}}\right)+q^{2}\left(\left(f_{3}+\frac{\phi_{2}}{t^{4} q^{3}}+\frac{\phi_{2}}{t^{3} q^{4}}+\frac{\phi_{1}}{t^{7} q^{7}}\right)\right. \\
\quad t\left(\phi_{3}+\frac{\phi_{2}}{t^{2} q^{4}}+\frac{\phi_{2}}{t^{4} q^{2}}+\frac{\phi_{1}}{t^{6} q^{6}}\right)+q\left(f_{3}+\frac{\phi_{2}}{t^{2} q^{4}}+\frac{\phi_{2}}{t^{4} q^{3}}+\frac{\phi_{1}}{t^{6} q^{7}}\right)+\left(\phi_{3}+\frac{\phi_{2}}{t^{2} q^{4}}+\frac{\phi_{2}}{t^{3} q^{3}}+\frac{\phi_{1}}{t^{5} q^{7}}\right) \\
=\left((1+t)(1+q)+t^{2}+q^{2}\right) \phi_{3}+ \\
+\left(t^{3} q+t^{2} q^{2}+t q^{3}+t^{2} q+q^{3}+q^{2} t+t^{3}+t q^{2}+q t^{2}+q^{2}+t^{2}+t q\right) \frac{\phi_{2}}{t^{4} q^{4}}+ \\
+\left(t^{2} q^{2}+t q^{2}+q^{2}+t^{2} q+t q+t^{2}\right) \frac{\phi_{1}}{t^{7} q^{7}} \\
=\left((1+t)(1+q)+t^{2}+q^{2}\right) \phi_{3}+\left(q^{2}(1+t+q+t q)+t^{2}(1+t+q+t q)+q t(1+t+q+t q)\right) \frac{\phi_{2}}{t^{4} q^{4}}+ \\
\quad+\left(t q(t q+q+t+1)+t^{2}+q^{2}\right) \frac{\phi_{1}}{t^{7} q^{7}} \\
\left((1+t)(1+q)+t^{2}+q^{2}\right) \phi_{3}+(1+t)(1+q)\left(t^{2}+t q+q^{2}\right) \frac{\phi_{2}}{t^{4} q^{4}}+\left(t q(1+t)(1+q)+t^{2}+q^{2}\right) \frac{\phi_{1}}{t^{7} q^{7}}
\end{gathered}
$$

This completes our proof of 5.10 . 
It turns out that 5.10 is but a particular case of the following result which may derived from Proposition 1.2 of the "Lattice Diagrams" paper [3].

\section{Theorem 5.1}

For a partition $\mu=\left(\mu_{1}, \mu_{2}, \ldots, \mu_{k}\right)$ with $m$ corners we have

$$
\partial_{p_{1}} \tilde{H}_{\mu}=\sum_{i=1}^{k} \sum_{j=1}^{\mu_{i}} t^{i-1} q^{j-1}\left(\sum_{\epsilon_{1}=0}^{1} \sum_{\epsilon_{2}=0}^{1} \cdots \sum_{\epsilon_{m}=0}^{1} \phi_{\epsilon_{1}, \epsilon_{2}, \ldots, \epsilon_{m}} \chi\left(j \leq \epsilon_{1} w_{1}+\epsilon_{2} w_{2}+\cdots \epsilon_{r_{i}} w_{r_{i}}\right)\right)
$$

where $w_{1}, w_{2}, \ldots, w_{m}$ are the lengths of the successive landings in the Ferrers diagram of $\lambda$ (see figure) and $r_{i}$ is the number of corners of $\mu$ that are weakly above row $i$.

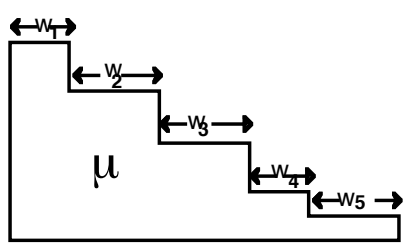

Note that we may view the summand in 5.25 as an assignment of modules $\mathbf{M}_{\epsilon_{1}, \epsilon_{2}, \ldots, \epsilon_{m}}$ to the cells of the diagram of $\mu$. Now this assigment in the case of $[3,2,1]$ reduces to

Cell $00 \rightarrow \sum_{\epsilon_{1}=0}^{1} \sum_{\epsilon_{2}=0}^{1} \sum_{\epsilon_{3}=0}^{1} \phi_{\epsilon_{1}, \epsilon_{2}, \epsilon_{m}} \chi\left(1 \leq \epsilon_{1}+\epsilon_{2}+\epsilon_{3}\right)=\phi_{001}+\phi_{010}+\phi_{011}+\phi_{100}+\phi_{101}+\phi_{110}+\phi_{111}$

Cell $01 \rightarrow \sum_{\epsilon_{1}=0}^{1} \sum_{\epsilon_{2}=0}^{1} \sum_{\epsilon_{3}=0}^{1} \phi_{\epsilon_{1}, \epsilon_{2}, \epsilon_{m}} \chi\left(2 \leq \epsilon_{1}+\epsilon_{2}+\epsilon_{3}\right)=\phi_{011}+\phi_{101}+\phi_{110}+\phi_{111}$

Cell $02 \rightarrow \sum_{\epsilon_{1}=0}^{1} \sum_{\epsilon_{2}=0}^{1} \sum_{\epsilon_{3}=0}^{1} \phi_{\epsilon_{1}, \epsilon_{2}, \epsilon_{m}} \chi\left(3 \leq \epsilon_{1}+\epsilon_{2}+\epsilon_{3}\right)=\phi_{111}$

Cell $10 \rightarrow \sum_{\epsilon_{1}=0}^{1} \sum_{\epsilon_{2}=0}^{1} \sum_{\epsilon_{3}=0}^{1} \phi_{\epsilon_{1}, \epsilon_{2}, \epsilon_{m}} \chi\left(1 \leq \epsilon_{1}+\epsilon_{2}\right)=\phi_{010}+\phi_{011}+\phi_{100}+\phi_{101}+\phi_{110}+\phi_{111}$

Cell $11 \rightarrow \sum_{\epsilon_{1}=0}^{1} \sum_{\epsilon_{2}=0}^{1} \sum_{\epsilon_{3}=0}^{1} \phi_{\epsilon_{1}, \epsilon_{2}, \epsilon_{m}} \chi\left(2 \leq \epsilon_{1}+\epsilon_{2}\right)=\phi_{110}+\phi_{111}$

Cell $20 \rightarrow \sum_{\epsilon_{1}=0}^{1} \sum_{\epsilon_{2}=0}^{1} \sum_{\epsilon_{3}=0}^{1} \phi_{\epsilon_{1}, \epsilon_{2}, \epsilon_{m}} \chi\left(1 \leq \epsilon_{1}\right)=\phi_{100}+\phi_{101}+\phi_{110}+\phi_{111}$

This assignment can be visualized as follows

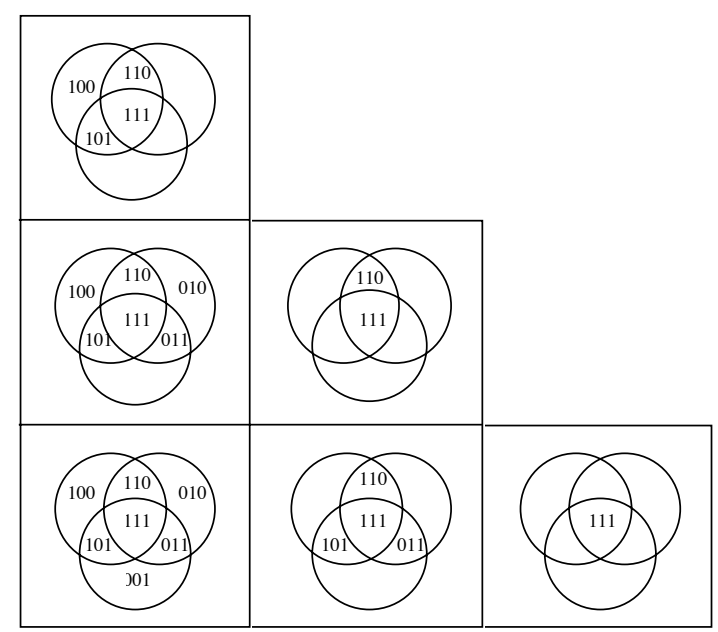




\section{REFERENCES}

[1] F. Bergeron and A. Garsia, Science Fiction and Macdonald Polynomials, Algebraic methods and $q$-special functions (J. P. Van Diejen, L. Vinet, ed.), CRM Proceedings \& Lecture Notes, American Mathematical Society, (1999), 1-52 .

[2] N. Bergeron and A. M. Garsia, On certain spaces of harmonic polynomials, Hypergeometric functions on domains of positivity, Jack polynomials, and applications (Tampa, FL, 1991), Contemp. Math., 138, Amer. Math. Soc., Providence, RI, (1992), 51-85.

[3] F. Bergeron, N. Bergeron, A. M. Garsia, M. Haiman and G. Tesler, Lattice Diagram Polynomials and Extended pieri Rules, Adv. in Math., 142 (1999), 244-334.

[4] A. Garsia and M. Haiman, Orbit Harmonics and Graded Representations, Laboratoire de combinatoire et d'informatique mathématique, Université du Québec à Montréal, in preparation.

[5] A. M. Garsia and M. Haiman, A graded representation model for Macdonald's polynomials, Proc. Nat. Acad. Sci. U.S.A. 90 (1993), no. 8, 3607-3610.

[6] A. Garsia and M. Haiman, Factorizations of Pieri rules for Macdonald polynomials, Discrete Math. 139 (1995), no. 1-3, 219-256, Formal power series and algebraic combinatorics (Montreal, PQ, 1992).

[7] A. Garsia and M. Haiman, Some natural bigraded $S_{n}$-modules and q,t-Kostka coefficients, Electron. J. Combin. 3 (1996), no. 2, Research Paper 24, approx. 60 pp. (electronic), The Foata Festschrift, http://www.combinatorics.org/Volume_3/volume3_1.html\#R24 .

[8] A. M. Garsia and C. Procesi, On certain graded $S_{n}$-modules and the q-Kostka polynomials, Adv. Math. 94 (1992), 82-138.

[9] J. Haglund, M. Haiman and N. Loehr, A combinatorial formula for Macdonald polynomials, Jour. Amer. Math. Soc. 18 (2005), 735-761.

[10] M. Haiman, Hilbert Schemes, polygraphs, and the Macdonald positivity conjecture, J. Amer. Math. Soc. 14 (2001), 941-1005.

[11] L. Lapointe, A. Lascoux and J. Morse, Tableau atoms and a new Macdonald positivity conjecture, Duke Math. J. 116 (2003), 103-145.

[12] I. G. Macdonald, A new class of symmetric functions, Séminaire Lotharingien de Combinatoire, Publ. Inst. Rech. Math. Av., 372, Univ. Louis Pasteur, Strasbourg, 1988, pp. 131-171.

[13] I. G. Macdonald, Symmetric functions and Hall polynomials, second ed., Oxford Mathematical Monographs, The Clarendon Press Oxford University Press, New York, 1995, With contributions by A. Zelevinsky, Oxford Science Publications.

[14] J. Stembridge, Some particular entries in the two parameter Kotska matrix, Proc. of the Amer. Math. Soc., 121 (1994), 367-373.

[15] M. Yoo, Combinatorial formulas connected to Diagonal Harmonics and Mcdonald Polynomials, PhD Thesis, Univ. of Pennsylvania. 